Livraisons

d'Histoire

de l'Architecture

\section{Livraisons de l'histoire de l'architecture}

$36 \mid 2018$

Jean-Louis Pascal (2)

\title{
Les monuments commémoratifs
}

The memorials

Denkmäler

\section{Anne Richard-Bazire}

\section{OpenEdition}

Journals

Édition électronique

URL : http://journals.openedition.org//ha/1044

DOI : $10.4000 /$ /ha. 1044

ISSN : 1960-5994

Éditeur

Association Livraisons d'histoire de l'architecture - LHA

Édition imprimée

Date de publication : 15 décembre 2018

Pagination : 141-173

ISSN : $1627-4970$

Référence électronique

Anne Richard-Bazire, "Les monuments commémoratifs », Livraisons de l'histoire de l'architecture [En ligne], 36 | 2018, mis en ligne le 15 décembre 2020, consulté le 25 janvier 2021. URL : http:// journals.openedition.org//ha/1044; DOI : https://doi.org/10.4000//ha.1044 


\section{LES MONUMENTS COMMÉMORATIFS}

En trois décennies, Jean-Louis Pascal a édifié quatre monuments dédiés à des personnalités du XIX ${ }^{\mathrm{e}}$ siècle : peintre, écrivain, homme politique ou architecte ; raffinement du petit temple à l'antique du monument au peintre Henri Regnault dans la cour du mûrier de l'École des Beaux-Arts ${ }^{1}$, effervescence baroque du monument à Victor Hugo, place d'Eylau ${ }^{2}$ à Paris, sobriété du monument à Sadi Carnot à Bordeaux et enfin simplicité du buste de Charles Garnier devant l'Opéra de Paris, à chaque fois, Pascal s'est adapté à la personnalité du personnage et aux ressources dont il disposait.

Ces monuments sont aussi l'histoire d'amitiés entre artistes: Henri Chapu sculpte la très belle Jeunesse de marbre pour le peintre Henri Regnault fauché dans la fleur de l'âge, dont le buste est réalisé par Charles-Marie Degeorge ${ }^{3}$. Ernest Barrias appelle Pascal pour collaborer avec lui au monument à Sadi Carnot et à celui de Victor Hugo pour lequel le sculpteur Denys Puech, parent de Pascal par sa femme, réalise les médaillons de bronze, enfin le sculpteur Gabriel Thomas, ami d'enfance de Charles Garnier réalise pour le monument de l'Opéra les allégories de bronze qui accompagnent le buste de l'architecte réalisé par un autre de ses proches, JeanBaptiste Carpeaux ${ }^{4}$.

\section{Le monument à Victor Hugo (1885-1902)}

L'histoire de ce monument (ill. 1) est celle de dix-sept ans d'atermoiements entre Paul Meurice (1818-1905), exécuteur testamentaire de Victor Hugo et président du comité créé dès la mort de l'écrivain en vue de lui ériger un monument, et le sculpteur Ernest Barrias, chargé de son exécution. Lutte ouverte dont Jean-Louis Pascal, choisi comme architecte du monument, fut l'arbitre à son corps défendant.

1. Voir Anne Richard-Bazire, "Avant la bibliothèque ", Livraisons d'histoire de l'architecture, $\mathrm{n}^{\circ} 28$, 2014, p. 57-62.

2. Actuelle place Victor Hugo.

3. Henri Regnault et Charles-Marie Degeorge étaient pensionnaires de la Villa Médicis en même temps que Jean-Louis Pascal.

4. Charles Garnier dédicacera ainsi à J.-B. Carpeaux l'exemplaire de son ouvrage $\grave{A}$ travers les arts: "Veux-tu accepter ce petit livre, il te rappellera le souvenir d'un ami qui est tout fier d'avoir servi de modèle pour un de tes chefs-d'œuvre. "Bibl. Opéra, Fonds Garnier, pièce 122, liste des amis de Garnier annotée, 6 p. Ms., numérotées de 1 à 19. 


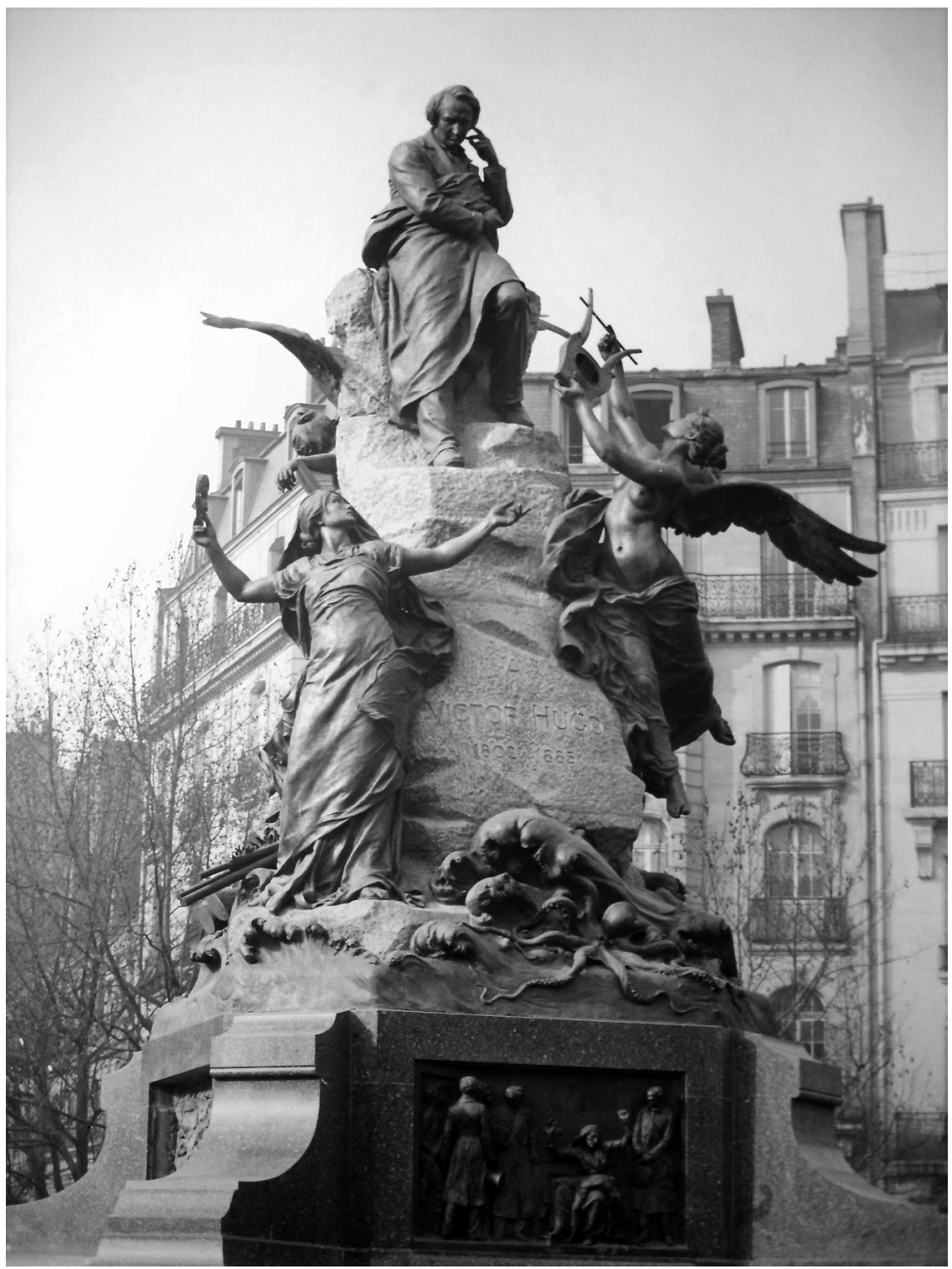

Ill. 1 : Monument à Victor Hugo. Architecte Jean-Louis Pascal. 1902. (Détruit en 1941). Ancienne place d'Eylau (actuelle place Victor Hugo), Paris $\left(16^{\mathrm{e}}\right)$. Granit et bronze. Sculpteur : Ernest Barrias : groupe principal de Victor Hugo et les muses (muse lyrique à droite, muse dramatique à gauche) et bas-relief de bronze: Souvenir de la nuit du quatre. Archives de la Maison de Victor Hugo. Dossier : Monuments I - Barrias. Photo 1916. 
C'est Ernest Barrias qui le choisit, après avoir demandé son avis à Charles Garnier qui faisait partie de la commission artistique ${ }^{5}$ destinée à juger la sculpture :

«M. Garnier est venu voir mon esquisse; il s'en est montré très satisfait. D'accord avec lui, j'ai chargé M. Pascal, architecte à la Bibliothèque nationale, membre de l'Institut, de faire un devis pour toute la partie architecturale du soubassement, avec bas-reliefs et deux médaillons et tout le rocher en granit ${ }^{6}$.

La notoriété d'un membre de l'Institut, architecte de la Bibliothèque nationale, mais surtout une longue amitié remontant à la Villa Médicis et la collaboration sur de nombreux chantiers ${ }^{7}$ peuvent expliquer le choix de Jean-Louis Pascal par Ernest Barrias. Le monument sera réalisé après dix-sept ans de querelles entre les différents protagonistes de l'affaire et inauguré le 26 février $1902^{8}$. Cette statue monumentale 9 , érigée sur l'ancienne place d'Eylau ${ }^{10}$ - actuelle place Victor Hugo ${ }^{11}$ - à Paris, sera fondue en 1941. Il n'en subsiste que les bas-reliefs et un médaillon, insérés dans un mur de jardin public en $\operatorname{Normandie}^{12}$ (ill. 2).

5. Celle-ci était composée du président du comité : Paul Meurice, d'Émile Blémont, trésorier, de Léon Bonnat, de Charles Garnier et de Philippe Gille. Arch. de la Maison de Victor Hugo, dossier: Monuments I - Barrias (Correspondance Barrias, correspondance Allar, atelier), contrat entre Paul Meurice et Ernest Barrias, 28 juillet 1896.

6. Ibid., lettre d'Ernest Barrias à Émile Blémont, 1898.

7. Le chantier de la faculté de médecine et de pharmacie de Bordeaux (1876-1888; 1902-1922), la statue de Sadi Carnot à Bordeaux (1895), puis le chantier de la Bibliothèque nationale.

8. C'est-à-dire le lendemain de l'ouverture des fêtes organisées par la ville de Paris, du 25 février au 2 mars 1902, pour célébrer le centenaire de la naissance d'Hugo. Le mercredi 26 février, des cérémonies officielles se déroulèrent dans l'hôtel de Massa et l'on inaugura à 15 h, en présence du ministre de l'instruction publique et des Beaux-Arts Georges Leygues, le monument de Jean-Louis Pascal et d'Ernest Barrias sur la place d'Eylau. Arch. de la maison de Victor Hugo, dossier : Monuments I - Barrias (Invitations, inauguration, inauguration de la Maison de Victor Hugo). Un peu plus d'un an plus tard, le mardi 30 juin 1903, à 10 h, on inaugurait la maison de Victor Hugo (avec un musée, une bibliothèque, des archives) au $\mathrm{n}^{\circ} 6$ de la place des Vosges (Paris, $4^{\mathrm{e}}$ ) au deuxième étage de l'hôtel de Rohan-Guéménée, où Victor Hugo avait demeuré avec sa famille de 1832 à 1848 , avant l'exil.

9. «Monument de Victor Hugo », La Construction moderne, 1901-2, p. 281-282.

10. C’est dans sa séance du 5 avril 1881 que le conseil municipal de Paris proposa de rebaptiser non seulement la place d'Eylau (ainsi appelée de 1864 à 1885, puis appelée place Victor Hugo), mais aussi l'avenue qui la reliait au bois de Boulogne, pour lui donner le nom du poète. Arch. nat., FI CI 168, lettre du préfet de la Seine au ministre de l'intérieur et des cultes, 14 avril 1881, cité dans Chantal Martinet, "Les hommages publics ", La Gloire de Victor Hugo, Paris, Grand Palais, $1985-6$, p. $257-325$, note 43 , p. 295.

11. Pas très loin de l'hôtel particulier où Victor Hugo emménage en 1881, au $\mathrm{n}^{\circ} 130$ de l'avenue d'Eylau, actuel $n^{\circ} 124$ du boulevard Victor Hugo (16 $)$. Il y meurt le 22 mai 1885. La maison fut démolie en 1907. Jacques Hillairet, Dictionnaire historique des rues de Paris, Paris, éd. de Minuit, 1997, vol. 2, p. 633.

12. Au jardin public Saint Nicolas, à Veules-les-Roses (Seine-Maritime), petite station balnéaire que Paul Meurice avait fait découvrir à Victor Hugo, qui y fera trois séjours : 28 août-2 septembre 1879, 21 août-15 septembre 1881, septembre 1884. Voir Jean-Pierre Chaline, Victor Hugo et la Normandie, exposition du musée Victor Hugo de Villequier, juin-novembre 1985, Elizabeth Chirol (commissaire), ouvrage collectif, p. 252-253. C'est en souvenir de ces séjours que la ville de Paris 


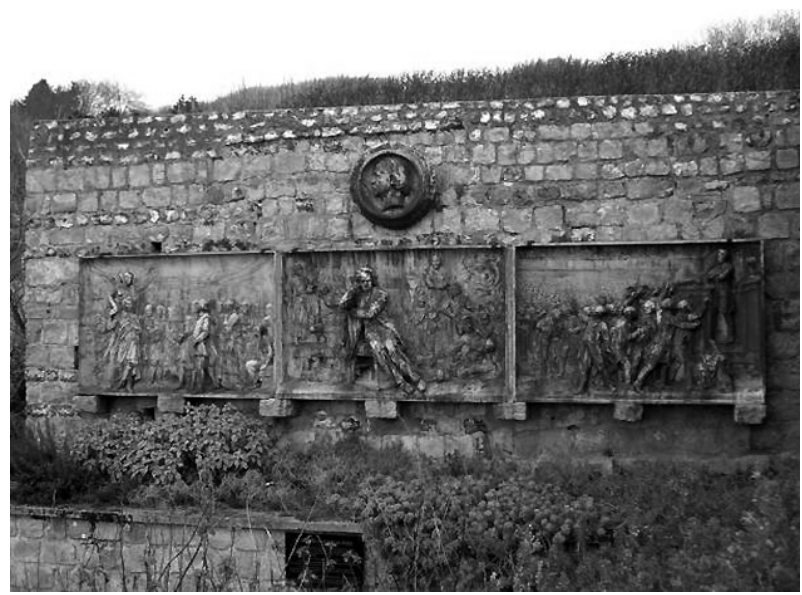

Ill. 2 : Trois bas-reliefs et le médaillon de bronze de Paul Meurice (Denys Puech) provenant du monument à Victor Hugo subsistent au jardin public Saint-Nicolas, à Veules-les-Roses (Seine-Maritime) : Victor Hugo reçu au Parnasse (André Allar), Victor Hugo et ses personnages (Ernest Barrias), Victor Hugo à la tribune, 17 juillet 1851 (André Allar). Cl. auteur.

Avant la mort de l'écrivain, un premier comité s'était réuni à Paris, le 5 juin 1881, à l'initiative de Louis Blanc (1811-1882), pour élever un monument au grand homme ${ }^{13}$. Cette première souscription, adressée à l'ensemble des conseils généraux fut un échec ${ }^{14}$. Il y avait deux types de motifs à ce refus : financiers ${ }^{15}$ (les plus nombreux) et politiques. Les conseils généraux à majorité réactionnaire, comme ceux de Vendée ${ }^{16}$ ou des Côtes-du-Nord ${ }^{17}$, refusèrent d'ériger une statue

a consenti au dépôt, par la petite-fille de Paul Meurice : Mme Langlois-Berthelot née Clémenceau, de trois bas-reliefs (Victor Hugo reçu au Parnasse, Victor Hugo et ses personnages, Victor Hugo à la tribune, 17 juillet 1851) et d'un médaillon (celui de Paul Meurice), à la commune; les bas-reliefs, plaques de bronze pesant entre 400 et $600 \mathrm{~kg}$ chacune, ont été posées par les entreprises de G. Legay, maçon et J. Grandsire, serrurier à Veules, sur les murs de soutènement du jardin Saint Nicolas. Un quatrième bas-relief (Souvenir de la nuit du quatre) a été déposé au musée des BeauxArts et de la dentelle de Calais par la ville de Paris. Arch. de la maison de Victor Hugo, dossier : Monument Barrias, coupure de journal : "Veules-les-Roses. Un médaillon et des bas-reliefs, qui ornaient l'ancien monument de Victor Hugo, à Paris, ont été posés au jardin Saint Nicolas ", et lettre de Martine Ecalle, conservateur en chef de la maison de Victor Hugo à Mme Langlois, 22 mai 1973.

13. Chantal Martinet, "Les hommages publics ", La Gloire de Victor Hugo, op. cit., p. 273.

14. Il y eut 28 réponses positives et 57 réponses négatives. Arch. de la maison de Victor Hugo, dossier : Monuments I - Souscription des conseils généraux, 1882.

15. Ce fut le cas par exemple de la préfecture du Lot : "Le conseil rejette, avec regrets, faute de fonds, la demande présentée dans le but d'obtenir une subvention pour l'érection d'une statue à Victor Hugo ", extrait du registre des délibérations du conseil général, séance du 19 avril 1882, ibid., et de celle du Finistère : lettre du préfet à Philippe Jourde, trésorier du comité, 3 mai 1882. Ibid.

16. "Je suis chargé par M. le Préfet en tournée de révision de vous faire connaître qu’à son grand regret, et d'après l'avis de la minorité républicaine au conseil général, il n'a pu soumettre à cette assemblée la proposition de souscrire pour la statue de Victor Hugo ; cette proposition eût été 
à un républicain. La réception de l'image du grand homme - poète ou homme politique - brouillait d'autre part les choses : "Victor Hugo trop ou pas assez idéologique, voilà ce qui freine les hommages en ces années [...] qui sont celles des luttes menées pour l'établissement définitif de la République " ${ }^{18}$. La préfecture de la Charente, elle, "laissait à l'État et à l'initiative privée le soin d'élever des statues aux grands hommes ${ }^{19}$. Le fait que Victor Hugo fût vivant gênait d'autre part sa "statufication ${ }^{20}$. En 1885, année de la mort de l'écrivain, un nouveau comité, présidé par Paul Meurice et Victor Schoelcher (1804-1893), fut créé21. Il lança une nouvelle souscription ${ }^{22}$ pour élever un monument à Victor Hugo ; on récolta tout d'abord la somme de cent dix mille francs, ce qui n'était pas suffisant pour le monument colossal que méritait Hugo. Paul Meurice et Auguste Vacquerie (1819-1895) étant chargés par le testament de Victor Hugo de revoir les manuscrits du poète et d'en assurer la publication, recevaient en échange de ce travail une part des droits d'auteur. Les deux amis de Victor Hugo abandonnèrent cette part de droits en faveur de la souscription au monument et, chaque année, plusieurs milliers de francs dus à leur générosité vinrent grossir la somme initiale. D’autre part, le trésorier de la souscription, Philippe Jourde, avait heureusement placé les cent dix mille francs versés, grâce à des bons du Trésor qui constituaient un excellent revenu à $5 \%$ (cent dix mille francs placés pendant quinze ans rapportèrent près de cent mille francs). Enfin Paul Meurice fit vendre à la maison Rouff un droit important de reproduction des ouvres d'Hugo et la famille ajouta spontanément une souscription de trente mille francs au capital en caisse ${ }^{23}$. Avec les dons de la famille et les

rejetée par la majorité réactionnaire compacte du conseil général de la Vendée ». Préfecture de la Vendée, La Roche-sur-Yon, lettre du 9 mai 1882 du secrétaire particulier du préfet au trésorier du comité. Ibid.

17. "Je m'empresse de vous faire connaître que le conseil général réactionnaire des Côtes-du-Nord auquel le comité pour l'érection d'une statue à Victor Hugo a adressé une demande de souscription, n'a voté aucun crédit et a même refusé d'examiner la question qui lui a été soumise ». Saint-Brieuc, lettre du 8 mai 1882 du cabinet du préfet des Côtes-du-Nord à Philippe Jourde. Ibid.

18. Chantal Martinet, "Les hommages publics ", La Gloire de Victor Hugo, op. cit., p. 276. Voir aussi Jean-Pierre Épron, Comprendre l'Éclectisme, Paris, éd. Norma, 1997, p. 192-226.

19. Angoulême, le 9 mai 1882, lettre du préfet au trésorier du comité. Arch. de la maison de Victor Hugo, dossier : Monuments I - Souscription des conseils généraux, 1882.

20. "J'ai l'honneur de vous informer que l'assemblée départementale, tout en rendant hommage au talent de M. Victor Hugo, n'a pas cru devoir voter une subvention pour élever une statue à un homme vivant ». Ce fut la raison donnée par le préfet de Seine-et-Marne pour expliquer le refus du conseil général de voter la souscription. 4 mai 1882. Ibid.

21. Comité d'exécution : présidents : Victor Schoelcher, Paul Meurice, vice-présidents : Émile Augier, Léon Bonnat, Anatole de la Forge, Auguste Vitu, secrétaires : Émile Blémont, Gustave Ollendorff, Gustave Rivet, trésorier : Philippe Jourde. Membres du comité provisoire et de la commission exécutive : président : Louis Blanc, membres : Edmond Bazire, Jules Clarétie, Armand Gonzien, Édouard Lebey, Édouard Lockroy, Paul Mantz, Marmottan, Catulle Mendès, Michelin, Louis Ulbach.

22. Circulaire en date du 29 juin 1885. Chantal Martinet, "Les hommages publics ", La Gloire de Victor Hugo, op. cit., note 99, p. 296.

23. Arch. de la maison de Victor Hugo, dossier : Monuments I - Barrias (Presse), «Victor Hugo par M. Barrias ", Le Temps, 18 janvier 1902. 
intérêts accumulés sur la souscription originale, la somme de trois cent mille francs fut atteinte ${ }^{24}$.

Un concours fermé ${ }^{25}$ fut organisé à l'issue duquel Ernest Barrias ${ }^{26}$ fut choisi pour le groupe principal et Jean Joseph Alexandre Falguière ${ }^{27}$, pour les bas-reliefs ${ }^{28}$. Pascal aurait préféré, par souci d'unité, que la totalité de la sculpture fût confiée à Barrias :

"Il serait pourtant bien nécessaire qu'un entente complète me permît de prendre la direction technique de l'exécution définitive [...] Falguière n'avait pas commencé les bas-reliefs desquels il m'avait dit qu'il consentirait à se charger pourvu qu'on le laissât faire ce qu'il voudrait [...] Il s'agit maintenant de décider si on les fera et dans ce cas à qui on les confere. Mon opinion est très formelle à cet égard : le monument gagnera en unité s'il est de la même main et la maîtrise dont a fait preuve Barrias dans cette grande conception se pliera à l'exécution nerveuse nécessaire aux bas-reliefs de bronze " 29 .

Jean-Louis Pascal proposait d'autre part que les grandes figures ainsi que les ornements, motifs symboliques : cuirasse, armes, pieuvre, flots (ill. 1 et 3), se détachassent en or sur le fond de granit; Ernest Barrias penchait pour une exécution toute en bronze si les figures n'étaient pas dorées ${ }^{30}$. Pascal le raconte à Louise Garnier quelques années plus tard :

24. June Hargrove, Les Statues de Paris, la représentation des grands hommes dans les rues et sur les places de Paris, Paris, Albin Michel, 1989, p. 169. C'était le deuxième monument le plus cher après celui de Léon Gambetta (1838-1882), (350 000 fr.), réalisé par l'architecte Louis-Charles Boileau (18371910) et le sculpteur Jean-Paul Aubé (1837-1916) en bronze et marbre. Inauguré sur la place du Carrousel à Paris, le 14 juillet 1888, il fut partiellement détruit en 1942.

25. "Un concours public aurait écarté les grands sculpteurs, mais ils acceptaient un concours à condition toutefois que ce concours fut tenu secret ", lettre échangée entre Paul Meurice et Catulle Mendès, membre du comité, septembre 1890, citée dans Chantal Martinet, "Les hommages publics », La gloire de Victor Hugo, op. cit., note 102, p. 196.

26. Arch. de la maison de Victor Hugo, dossier : Monuments I - Barrias (Correspondance Barrias, correspondance Allar, atelier), contrat passé entre le comité et Ernest Barrias, 28 juillet 1896, qui définit le programme de la sculpture et les conditions de son exécution.

27. Élève de Jouffroy à l'École des Beaux-Arts, il connaît une carrière pleine de succès et prend la suite de son maître comme professeur à l'École des Beaux-Arts (1882) et à l'Institut (1883). D'importants monuments publics lui ont été commandés en France (L'Asie, 1878, musée d'Orsay ; Monument à Pasteur, 1900, place de Breteuil, Paris). Il réalise d'émouvantes figures telle Tarcissius, 1867, musée d'Orsay.

28. "Le comité voudrait élever le monument sur la place qui porte le nom de Victor Hugo, entre la maison où il est mort et l'Arc de triomphe d'où il est parti pour aller au Panthéon. L'exécution est confiée à Barrias pour la sculpture et à Falguière pour les bas-reliefs ». Arch. de la maison de Victor Hugo, dossier : Monuments I - Barrias (Correspondance Barrias, correspondance Allar, atelier), lettre du 21 novembre 1896 de Paul Meurice au conseil municipal de Paris.

29. Arch. de la maison de Victor Hugo, dossier : Monuments I - Barrias (Localisation du monument, correspondance Pascal, affaire de la palissade), lettre de Jean-Louis Pascal à Paul Meurice, Paris, le 22 mai 1900.

30. Ibid. 


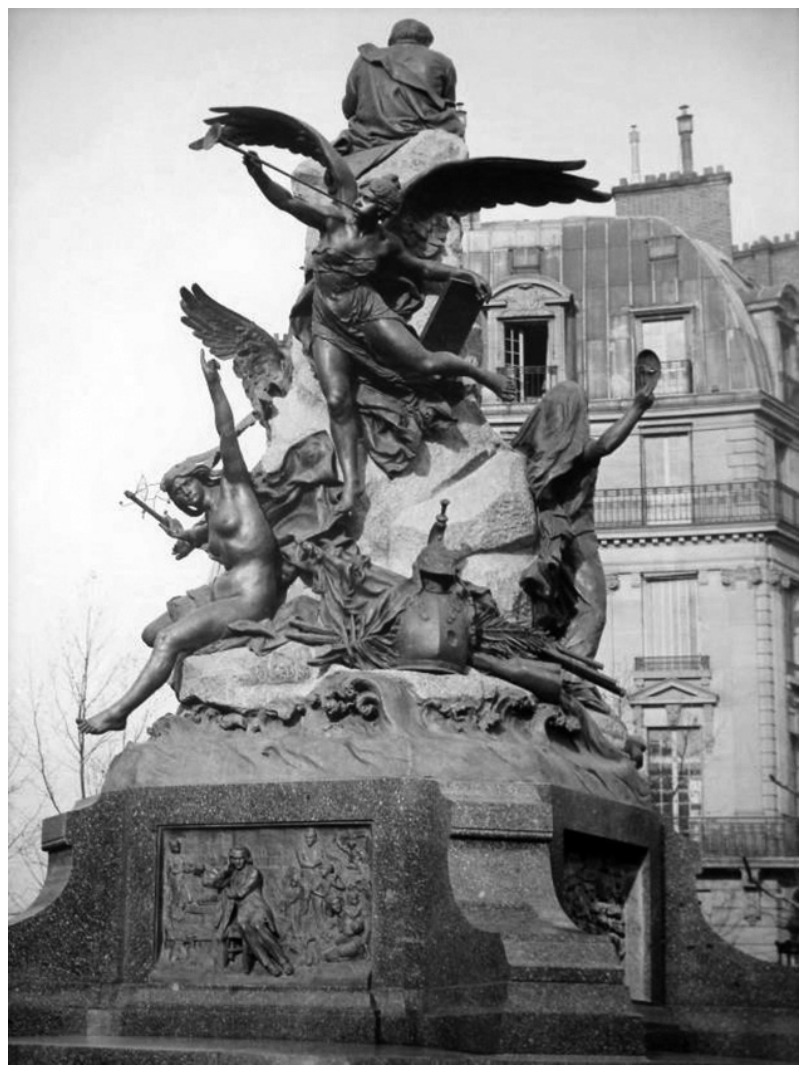

Ill. 3 : Arrière du monument à Victor Hugo. Architecte Jean-Louis Pascal. 1902. (Détruit en 1941). Ancienne place d'Eylau (actuelle place Victor Hugo), Paris $\left(16^{e}\right)$. Granit et bronze. Sculpteur : Ernest Barrias : groupe principal de Victor Hugo et les muses (muse épique en bas à gauche, muse satirique au centre sous la statue de Hugo) et bas-relief de bronze : Victor Hugo et ses personnages. Archives de la Maison de Victor Hugo. Dossier : Monuments I - Barrias. Photo datée de 1916.

«Avec Barrias nous voulions dorer le monument Victor Hugo et nous en avons même fait l'essai complet pour la Poésie lyrique. La famille et le comité y ont fait objection et nous avons déjà [nous sommes alors en 1905] les bronzes tout noirs comme il arrive de tous les bronzes à Paris, bien que nous ne les eussions pas fait patiner et que le désir de Barrias fût qu'ils s'enlevassent en clair sur le granit ${ }^{31}$.»

\section{Polémique sur le programme}

Des désaccords multiples se produisirent entre Ernest Barrias et Paul Meurice et le jour de l'inauguration, les deux hommes ne se parlaient plus. Au début, les rapports étaient cordiaux. Voici ce qu'écrit le sculpteur à Paul Meurice, le 2 juillet 1896 :

31. Bibliothèque de l'École des Beaux-Arts. (B.E.B.A)., Ms 745, Lettres de l'architecte Jean-Louis Pascal à Louise Garnier, lettre ${ }^{\circ}$ 18, Paris, 26 mars 1905. 
"Pensez bien, mon cher Paul Meurice, que vous êtes l'âme du monument [...] Croyez-en mon expérience, ne laissez rien faire à un comité, faites tout par vous-même ; ne laissons pas discuter l'œuvre sur un programme en plâtre, objet de critiques intéressées, qui pourrait provoquer de nouvelles convoitises, et, faire reporter bien loin après 1902 l'exécution définitive [...] $\mathrm{Ne}$ laissons discuter non seulement l'œuvre, mais même le programme, sous peine de remaniements sans fin. Le programme, vous l'avez longuement médité avec M. Vacquerie. Les critiques d'une autre génération demanderont: "Pourquoi ceci ? Pourquoi cela ?" Certaines pensées ne sont plus comprises à vingt ans de distance ; ainsi on me demande déjà : "Pourquoi Victor Hugo sur un rocher? Que nous importe qu'il ait été exilé ? Nous ne rendons hommage qu'au poète!" Ceux-là sont des jeunes qui n'ont pas connu le 2 décembre et l'exil " ${ }^{32}$.

Le contrat entre Paul Meurice et Ernest Barrias ${ }^{33}$ qui date du 28 juillet 1896, fixait l'iconographie. Le monument représentait un rocher en granit simulant l'île rocheuse de Guernesey et supportant la statue d'un Victor Hugo ${ }^{34}$ jeune et rêveur. Sur ce rocher étaient diversement placées quatre figures en bronze ${ }^{35}$ représentant les quatre modes créatifs de l'écrivain : la Muse lyrique, la Muse dramatique, la Muse épique et la Muse satirique, inspirées des Quatre vents de l'esprit. Les vagues mugissantes déversaient à ses pieds une récolte abondante d'attributs littéraires, de trophées, ainsi qu'une pieuvre, symboles de son œuvre prolifique. Dans un texte intitulé Le monument de Victor Hugo ${ }^{36}$, Paul Meurice expose ses vues :

"Ce qui caractérise essentiellement le génie poétique de Victor Hugo, c’est qu'il a été grand à la fois sous les différentes formes et les plus hautes de la poésie : l'Ode, le Drame, l'Épopée et la Satire. Dans Les Feuilles d'automne

32. Arch. de la maison de Victor Hugo, dossier: Monuments I - Barrias (Correspondance Barrias, correspondance Allar, atelier), lettre d'Ernest Barrias à Paul Meurice, 2 juillet 1896.

33. Les paiements se faisaient ainsi :

- première année : à la signature du contrat : $5000 \mathrm{fr}$.

le petit modèle ébauché : $5000 \mathrm{fr}$.

le même complètement terminé : $10000 \mathrm{fr}$.

- deuxième année : 25000 fr.

- troisième année et quatrième année : 35000 fr.

- cinquième et sixième années : 119000 fr.

- bas-reliefs et médaillons : $26000 \mathrm{fr}$.

Prix total : 225000 fr. Les fondations étaient à la charge d'Ernest Barrias.

34. Le portrait d'Hugo s'inspire de photos prises à Guernesey par son fils alors que le poète exilé méditait sur les rochers. June Hargrove, Les Statues de Paris, la représentation des grands hommes dans les rues et sur les places de Paris, op. cit., p. 235.

35. "Les figures seront d'une proportion de trois mètres et reliées par des accessoires en bronze, trophées, vagues, etc. La statue de Victor Hugo sera plus grande [quatre mètres], suivant les nécessités de l'art décoratif et de la perspective ", contrat du 28 juillet 1896, archives de la maison de Victor Hugo. La hauteur totale du monument était de onze mètres.

36. Arch. de la maison de Victor Hugo, dossier: Monuments I - Barrias (Correspondance Barrias, correspondance Allar, atelier). 
et dans Les Contemplations, il égale Pindare; dans La Légende des siècles, il égale Dante; dans Hernani et dans Ruy Blas il atteint Shakespeare, dans Les Châtiments, il dépasse Juvénal. Or ces génies n'ont guère de chef-d'œuvre que dans une seule forme d'art; Victor Hugo a les quatre couronnes".

Paul Meurice proposait de grouper autour de Victor Hugo les quatre muses; à droite du monument, la Muse lyrique, "d'un vol élégant et doux, lui apporterait la lyre qu'elle élèverait à deux mains vers lui ». À gauche, la Muse dramatique "à-demi tournée vers le poète, écarterait pour lui, le masque souriant de la comédie et lui découvrirait son visage tragique ». En arrière de Victor Hugo, sur le côté gauche, " la Renommée tiendrait de la main droite la trompette épique, tandis que la main gauche indiquerait que c'est de la part du poète qu'elle proclame les grandes actions des hommes " ${ }^{37}$. Sur le côté arrière droit du monument, "la Muse satirique agiterait de la main droite le fouet vengeur, et, du pouce de la main gauche, indiquerait le poète, comme si elle criait aux passions méchantes et viles : voilà la justice, prenez garde à vous! " ${ }^{38}$. Le modèle en est terminé au début du mois de janvier 1900 et repose dans les sous-sols du Grand Palais en attendant l'exposition universelle de $1900^{39}$. Le contrat stipulait «qu'un modèle teinté et colorié, grand d'exécution, devrait être achevé de façon à figurer à l'exposition universelle de 1900 ». Le 30 avril 1900, Ernest Barrias écrit à Paul Meurice : "Le monument de Victor Hugo est monté, sous le dôme de la grande nef du Grand Palais [...] demain [c'est] le jour de l'inauguration [...] Le monument a beaucoup de succès auprès des artistes. J'en espère moins auprès d'une certaine presse, dont l'intérêt n'est pas là " ${ }^{40}$. Jean-Louis Pascal est là, le jour de l'inauguration, poussant sa femme souffrante, dans un fauteuil roulant: "Nous avons beaucoup regretté le voisinage des énormes figures américaines dont on a encombré la nef au grand détriment des autres sculptures et d'elles-mêmes " ${ }^{41}$. C'est la maison Barbedienne ${ }^{42}$ qui assura la réalisation en bronze du monument (fonte au sable). La principale pièce de celui-ci fut coulée pendant la semaine du 10 au 17 mars $1901^{43}$; le 9 août 1901, Ernest Barrias est fier d'annoncer : "À la fin du présent mois, la plus grande partie de la fonte sera terminée et le granit, que j'ai été voir la semaine passée, dans les Vosges, est également très avancé " ${ }^{44}$. Le 21 août, le sculpteur écrit à Paul Meurice: "Dans quelques

37. La Renommée tient de sa main gauche une table où il est prévu de graver "La Légende des Siècles ".

38. Arch. de la maison de Victor Hugo, dossier: Monuments I - Barrias (Correspondance Barrias, correspondance Allar, atelier), texte de Paul Meurice intitulé : Le monument de Victor Hugo.

39. Ibid., lettre d'Ernest Barrias à Paul Meurice, 9 janvier 1900.

40. Ibid., lettre d'Ernest Barrias à Paul Meurice, 30 avril 1900.

41. Ibid., lettre de Jean-Louis Pascal à Paul Meurice, 22 mai 1900.

42. Maison Georges Leblanc-Barbedienne, 30 boulevard Poissonnière. Ateliers : 63, rue de Lassery.

43. Maurice Gérard, "Comment on coule une statue ", L'Actualité Française, Étrangère et Littéraire Illustrée, $2^{\mathrm{e}}$ année, $\mathrm{n}^{\circ}$ 60, dimanche 17 mars 1901. Arch. de la maison de Victor Hugo, dossier: Monuments I - Barrias (Invitations, inauguration, inauguration de la Maison de Victor Hugo).

44. Arch. de la maison de Victor Hugo, dossier: Monuments I - Barrias (Correspondance Barrias, correspondance Allar, atelier), lettre d'Ernest Barrias à Paul Meurice, 9 août 1901. 


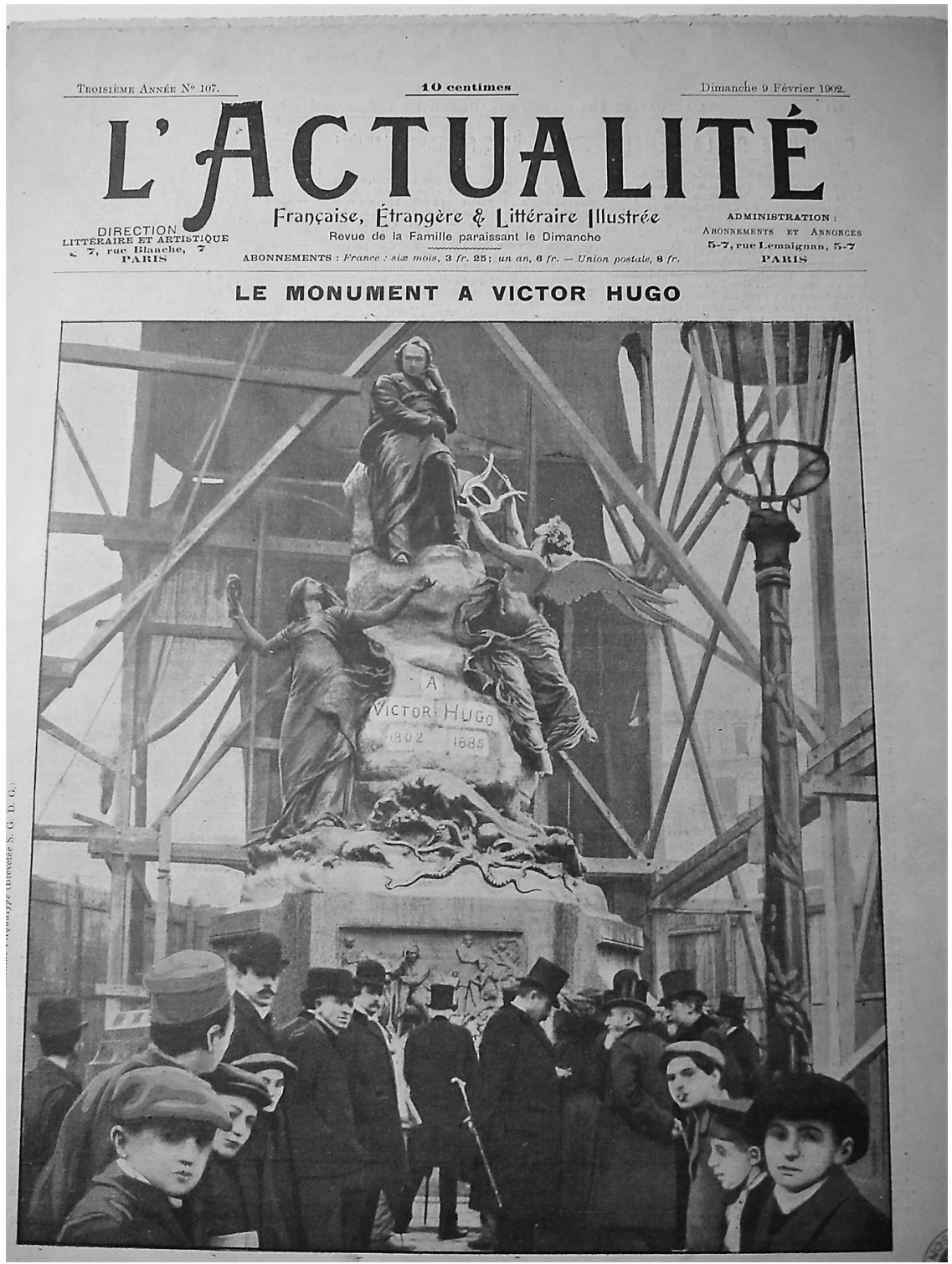

Ill. 4 : Monument à Victor Hugo. 1902. Ancienne place d'Eylau. 17 janvier 1902, jour du dévoilement au comité d'organisation. Ce cliché est intéressant car il montre la première version proposée par JeanLouis Pascal à Paul Meurice qui voulait placer à l'avant du monument le bas-relief de Victor Hugo et ses personnages, qu'il trouvait moins polémique que celui du Souvenir de la nuit du quatre. Ernest Barrias, lui, voulait placer Souvenir de la nuit du quatre devant le monument. C'est ce qui sera fait dans la version définitive. Archives de la Maison de Victor Hugo. Dossier : Monuments I - Barrias. "Le monument à Victor Hugo ", L'Actualité Française, Étrangère et Littéraire Illustrée, no 107, dimanche 9 février 1902. 
jours, on va poser les bronzes; il ne reste plus qu'à graver sur la table tenue par la Poésie épique. Nous avions primitivement gravé seulement La Légende des Siècles: faut-il graver d'autres titres? Lesquels? [Il y a] peu de place " ${ }^{45}$. Le 30 août, on place la grande figure de la Poésie dramatique ${ }^{46}$. Le 17 janvier 1902, le monument est dévoilé, le temps d'une visite, au comité d'organisation (ill. 4). Le 3 février 1902, le monument est terminé : "Il ne reste plus qu'à graver le nom et à le dorer " ${ }^{4}$. Jean-Louis Pascal proposa ${ }^{48}$ de demander au directeur des Beaux-Arts Henri Roujon, de faire décorer des palmes académiques le praticien sculpteur Tessier ${ }^{49}$, qui avait dirigé toute l'opération sur place, et le chef de fabrication de la maison LeblancBarbedienne, Bellenot ${ }^{50}$.

L'ensemble du monument reposait sur un soubassement en granit dans lequel devaient être incrustés les quatre bas-reliefs ${ }^{51}$. Si « les muses guident le spectateur dans le monde imaginaire du poète, leur beauté idéalisée les séparant de la réalité physique, dans les bas-reliefs, la combinaison est renversée. La personne réelle de Hugo empiète dans le domaine fictif de la littérature " ${ }^{52}$. Ernest Barrias en demanda le programme à Paul Meurice ${ }^{53}$. Le modèle d'un premier bas-relief : Victor Hugo

45. Ibid., lettre d'Ernest Barrias à Paul Meurice, 21 août 1901.

46. Ibid., lettre d'Ernest Barrias à Paul Meurice, 30 août 1901.

47. Ibid., lettre d'Ernest Barrias à Paul Meurice, 3 février 1902.

48. Arch. de la maison de Victor Hugo, dossier Monuments I - Barrias (Localisation du monument, correspondance Pascal, affaire de la palissade), pneumatiques de Jean-Louis Pascal à Paul Meurice, 16 février et 15 mars 1902.

49. Sis 41 rue Liancourt, Paris, $14^{\mathrm{e}}$.

50. 26 avenue Paul Dupont à Gargan (Seine-et-Oise). Les autres collaborateurs au monument étaient : - Langlet, metteur au point.

- La maison Barbedienne : Royet, Schlüter, Jandilion, Jactet, Plat, Legroux, Camali.

- La maison Leclercq et Bourdon, fournisseur de granit (60 rue Caumartin), avait pour sous-traitants :

- Maison Poirier, charpente : Blot;

- Maison Dior, maçonnerie : Miaut, Bellot, Duprat (les ouvriers bardeurs);

- Maison Étienne, granit, dans les Vosges : Roch, Py, Mongel ;

- Maison Autin, emballage, charpente : Rastel, Louis Fleuron ;

- Maison Philippon, moulage : Bouture;

- Maison Loynet, dorure : deux ouvriers ;

- Le dernier ouvrier granitier parisien : Caudelier, de la Maison Leclercq.

Jean-Louis Pascal proposa une répartition de 2000 fr. entre les collaborateurs au monument Victor Hugo. Arch. de la maison de Victor Hugo, dossier: Monuments I - Barrias (Correspondance Barrias, correspondance Allar, atelier), lettre de Jean-Louis Pascal à Paul Meurice, 19 mars 1902.

51. Trois bas-reliefs sont actuellement placés dans le jardin public de Veules-les-Roses (Seine-Maritime), ceux représentant: Victor Hugo reçu au Parnasse et Victor Hugo à la tribune, le 17 juillet 1851 par André Allar, ainsi que Victor Hugo et ses personnages par Ernest Barrias. Le quatrième bas-relief : Souvenir de la nuit du quatre, par Ernest Barrias, est conservé au Musée des Beaux-Arts et de la dentelle de Calais.

52. June Hargrove, Les Statues de Paris, la représentation des grands hommes dans les rues et sur les places de Paris, op. cit., p. 235.

53. "Vous seriez bien aimable de m'envoyer le plus tôt possible le programme des bas-reliefs du monument à Victor Hugo ». Arch. de la maison de Victor Hugo, dossier : Monuments I - Barrias (Correspondance Barrias, correspondance Allar, atelier), lettre d'Ernest Barrias à Paul Meurice, début de l'année 1900. 


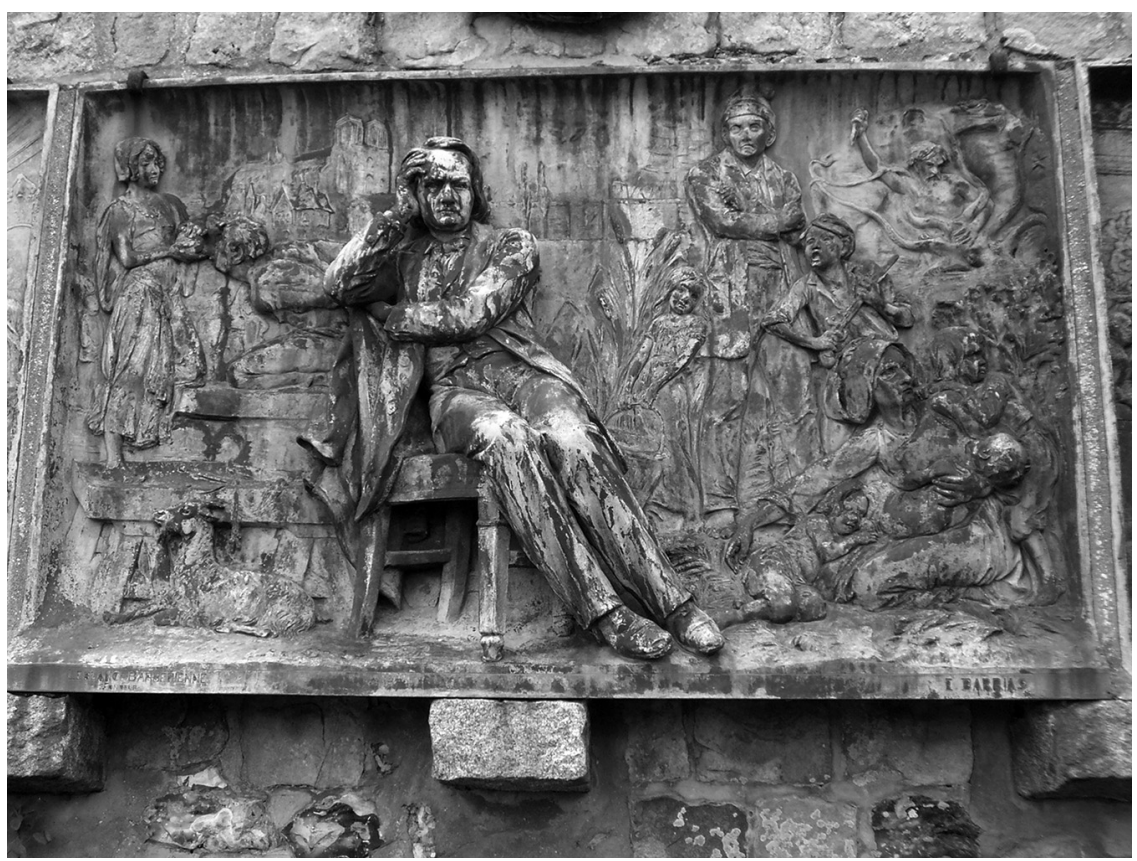

Ill. 5 : Bas-relief de bronze subsistant du monument à Victor Hugo au jardin Saint-Nicolas à Veulesles-Roses (Seine-Maritime). Victor Hugo et ses personnages (Ernest Barrias). Cl. auteur.

et ses personnages (ill. 5) étant achevé, Ernest Barrias invita le président du comité à venir le voir ${ }^{54}$. Puis, entre mars et juin 1901, il demanda plusieurs fois à Paul Meurice de venir lui donner son avis sur le deuxième relief qui lui avait été attribué, Souvenir de la nuit du quatre ${ }^{55}$ : "Je vous demanderai de venir voir le deuxième bas-relief, qui est très avancé; le premier est fondu, ainsi que presque tout le groupe ; il ne manque plus que deux bras pour que le tout soit terminé. Monsieur Allar, qui a bien voulu se charger des deux autres bas-reliefs, pourra vous montrer bientôt ses esquisses " ${ }^{56}$. En effet, Alexandre Falguière étant mort en 1900, Ernest Barrias et André Joseph Allar (1845-1926) furent chargés chacun de deux basreliefs ${ }^{57}$. Paul Meurice commença à être réticent dès janvier 1901: "J'ai été très

54. "Je vais faire mouler le bas-relief, pourriez-vous venir le voir ? ". Ibid., lettre d'Ernest Barrias à Paul Meurice, 30 juin 1900.

55. Illustrant le vers tiré des Châtiments : "L'enfant avait reçu deux balles dans la tête ». Deux jours après le coup d'état du 2 décembre 1851, les troupes gouvernementales ouvrent le feu sur la foule. Victor Hugo, qui avait essayé d'organiser la résistance, s'enfuit pour Bruxelles.

56. Arch. de la maison de Victor Hugo, dossier Monuments I - Barrias (Correspondance Barrias, correspondance Allar, atelier), lettre d'Ernest Barrias à Paul Meurice, 13 juin 1901. Une lettre précédente, datée du 3 mars 1901, enjoignait déjà Paul Meurice à venir voir le bas-relief.

57. Ernest Barrias réalise les bas-reliefs : Souvenir de la nuit du quatre et Victor Hugo et ses personnages. André Allar réalise: Victor Hugo reçu au Parnasse et Victor Hugo à la tribune, 17 juillet 1851. Les deux bas-reliefs dus à André Allar sont posés la dernière semaine du mois de février 1902 : «Lundi 


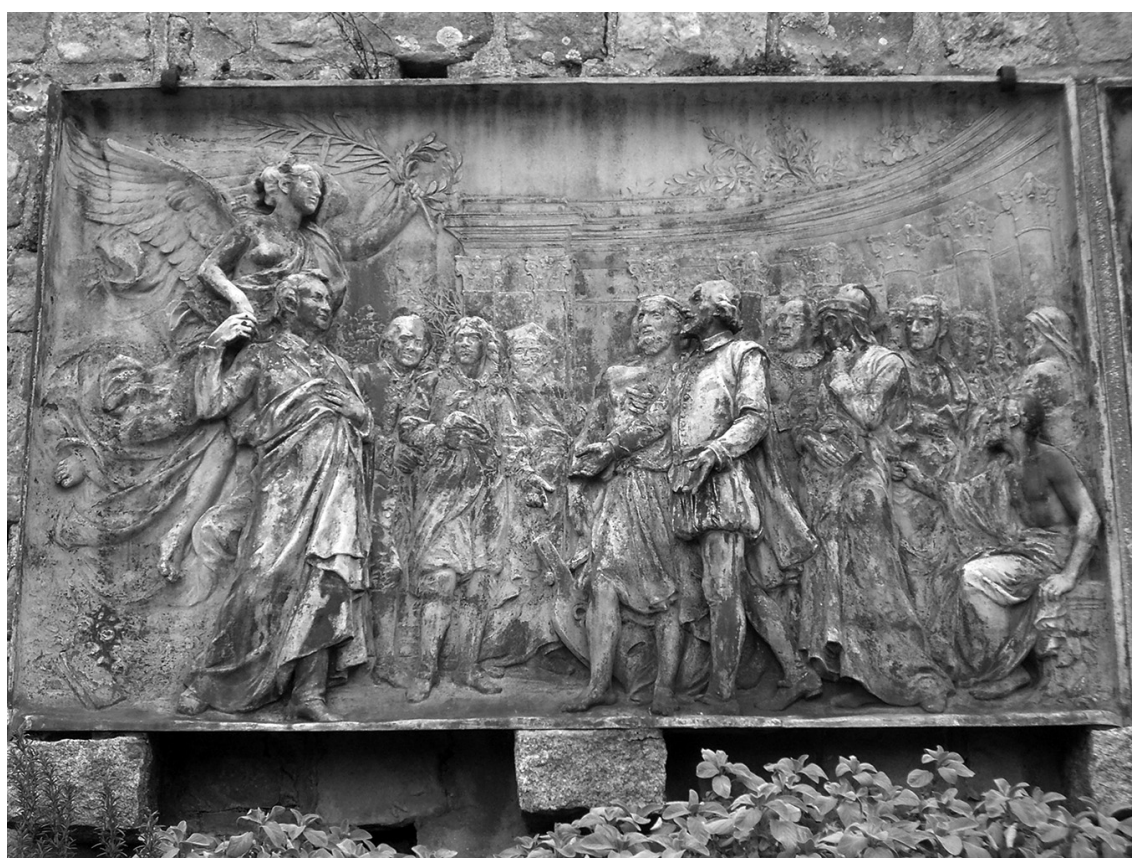

Ill. 6 : Bas-relief de bronze subsistant du monument à Victor Hugo au jardin Saint-Nicolas à Veulesles-Roses (Seine-Maritime). Victor Hugo reçu au Parnasse (André Allar). Cl. auteur.

surpris hier, lors de votre visite à mon atelier, [écrit Ernest Barrias] des hésitations que vous m'avez manifestées au sujet du comité et de son approbation à la commande des bas-reliefs " ${ }^{58}$. En effet, Ernest Barrias va orienter le monument, non seulement vers la glorification du poète avec Victor Hugo et ses personnages ${ }^{59}$ et Victor Hugo reçu au Parnasse ${ }^{60}$ (ill. 6), mais surtout vers celle de l'homme politique, du tribun avec Victor Hugo à la tribune, 17 juillet $1851^{61}$ (ill. 7) et vers celle du résistant à l'Empire avec Souvenir de la nuit du quatre (ill. 1), en mettant en exergue notamment ce

matin j'ai rendez-vous avec les ouvriers de M. Barbedienne pour la mise en place des deux basreliefs de Victor Hugo, celui qui le représente aux Champs-Élysées sera placé à gauche du monument et celui qui le représente pendant la séance mémorable du 17 juillet 1851 à droite ". Arch. de la maison de Victor Hugo, dossier Monuments I - Barrias (Correspondance Barrias, correspondance Allar, atelier), lettre d'André Allar à Paul Meurice, 22 février 1902. Le prix des bas-reliefs était de $6000 \mathrm{fr}$. chacun et celui des médaillons de $1000 \mathrm{fr}$. chacun (fonte, patine et pose comprise) soit un total de 26000 fr. Ibid., lettre d'Ernest Barrias à Paul Meurice, 8 février 1896.

58. Ibid., lettre d'Ernest Barrias à Paul Meurice, 21 janvier 1901.

59. Victor Hugo est entouré des personnages de ses romans : Esmeralda et Quasimodo, la Flécharde et ses enfants, Jean Valjean, Gavroche et Cosette, Gilliat et la pieuvre.

60. Victor Hugo est reçu au Parnasse par Homère, Dante, Ronsard, Molière.

61. Écarté du nouveau gouvernement par le Prince-Président, qu'il avait pourtant contribué à faite élire, Victor Hugo dénonce à la tribune de l'assemblée, le 17 juillet 1851, le cléricalisme du nouveau gouvernement et les ambitions dictatoriales de "Napoléon-le-Petit ". 


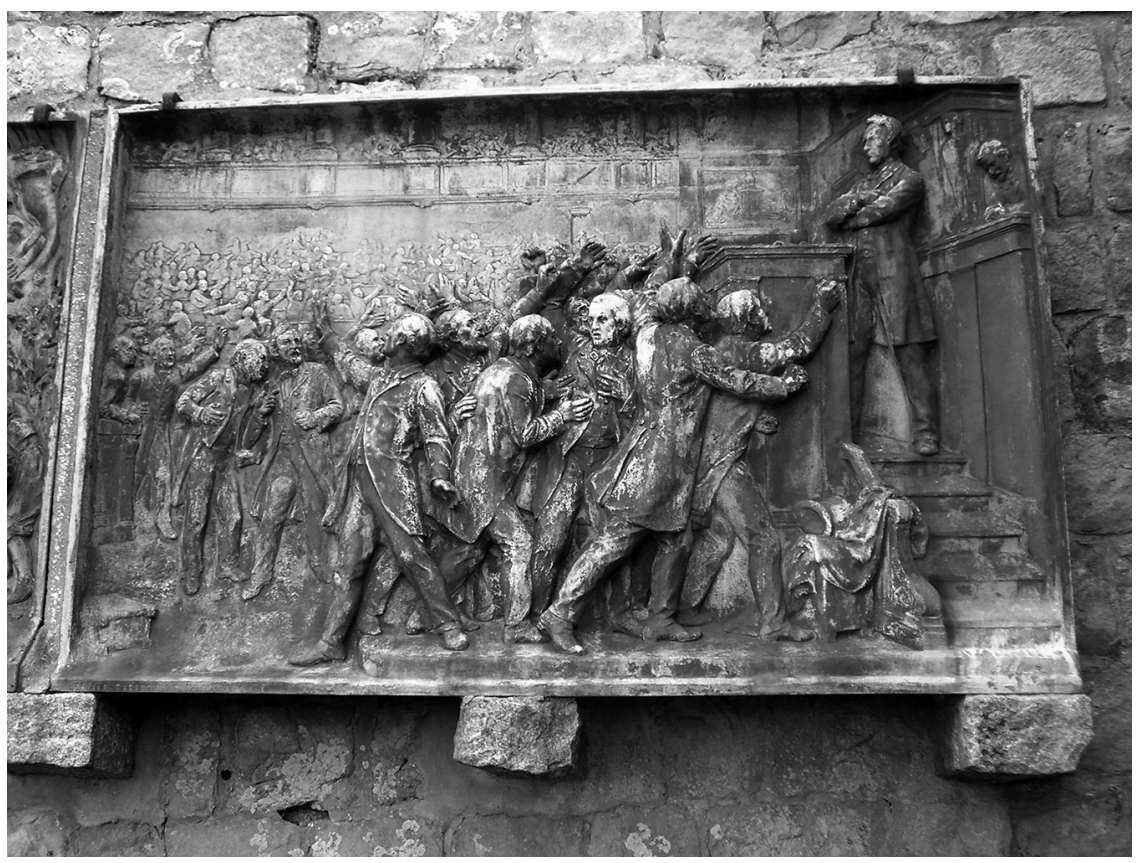

Ill. 7 : Bas-reliefs de bronze subsistant du monument à Victor Hugo au jardin Saint-Nicolas à Veulesles-Roses (Seine-Maritime). Victor Hugo à la tribune, 17 juillet 1851 (André Allar). Cl. auteur.

dernier, ce qui n'était pas du goût du comité. En plus des bas-reliefs, et pour compenser les sacrifices faits par le comité, on décida d'ajouter les portraits en médaillon de Paul Meurice par Denys Puech (1854-1942) et d'Auguste Vacquerie par Henri Chapu : "Je vous serais obligé [écrit Barrias à Meurice] de remettre le médaillon de M. Vacquerie par Chapu au porteur. Je le ferai grandir au plus [en] grandeur naturelle [...] On mettra ces deux médaillons à la place que vous indiquerez " ${ }^{62}$. Jean-Louis Pascal et Ernest Barrias proposèrent deux emplacements à gauche et à droite du rocher, mais cela nécessitait de réduire la taille des médaillons ${ }^{63}$. Estimant son portrait mal placé et trop petit, Paul Meurice renonça à placer des médaillons ${ }^{64}$. Jean-Louis Pascal en fut désolé : "Personnellement je n’admettrais guère que votre générosité fût le prix de l'abandon de l'introduction dans le monument, et, pour moi, dans le rocher, des médaillons de Vacquerie et du vôtre " ${ }^{65}$.

62. Arch. de la maison de Victor Hugo, dossier Monuments I - Barrias (Correspondance Barrias, correspondance Allar, atelier), lettre d'Ernest Barrias à Paul Meurice, 17 novembre 1901.

63. "Nous avons examiné avec Pascal la question des médaillons, et nous avons trouvé deux places sur le rocher, une place côté gauche, une autre côté droit, à la condition que ces médaillons soient plus petits ». Ibid., lettre d'Ernest Barrias à Paul Meurice, janvier 1902.

64. Ibid., lettre de Paul Meurice à Ernest Barrias, 15 février 1902.

65. Ibid., lettre de Jean-Louis Pascal à Paul Meurice, 19 mars 1902. 
Il fallut attendre le 20 juin $1910^{66}$ pour que les deux médaillons, finalement réalisés, ainsi que ceux ${ }^{67}$ des deux fils de Victor Hugo, Charles et François Victor ${ }^{68}$, morts prématurément, que l'on avait décidé d'ajouter, fussent inaugurés. Puis Ernest Barrias et Paul Meurice se chamaillèrent à propos du bas-relief : Le souvenir de la nuit $d u$ quatre, car Paul Meurice voulait qu'il y ait plus de "témoins" à La nuit du quatre. Ernest Barrias trouvait que cela surchargeait le bas-relief ${ }^{69}$. Pour Paul Meurice, cette scène évoquait des souvenirs désagréables de guerre civile; il aurait préféré y substituer, sur la face la plus en vue du monument, "une évocation des régions plus sereines de la création littéraire " ${ }^{70}$, c'est-à-dire le bas-relief de Victor Hugo au milieu de ses œuvres. Jean-Louis Pascal tenta de temporiser en écrivant à Paul Meurice :

«Nous [avec Barrias] avons constaté absolument que la répétition du motif du poète songeur dans le bas-relief et sur le rocher ${ }^{71}$, voire de la pieuvre à deux mètres de distance, et surtout que la composition très douce sur le fond, du créateur au milieu des œuvres, s'arrangeaient mal avec la partie haute du monument, tandis que d'autres faces s'en accommodaient très bien. Inutile de dire que votre argument de la logique de la synthèse générale qui appelait sur la face le sujet le plus typique - dans la grandiose carrière - était frappant et difficile à combattre ; que votre reproche du choix d'un sujet anecdotique comme : "l'enfant avait deux balles dans la tête", était fondé. Aussi avons-nous cherché à prendre, par exemple, le Victor Hugo reçu par les poètes. Mais le nombre des figures a obligé à diminuer leur échelle dans ce bas-relief de notre ami Allar. Il ne va pas aussi bien sur la face. Ce n'est pas à un artiste tel que vous qu'il faut expliquer que logique, conventions, raisonnement disparaissent devant la raison de beauté dans les œuvres d'art et c'est une pure raison de beauté plastique que j’invoque pour réclamer

66. June Hargrove, Les Statues de Paris, la représentation des grands hommes dans les rues et sur les places de Paris, op. cit., note 158, p. 366.

67. Ces deux médaillons furent réalisés par Denys Puech.

68. Charles, le fils aîné de Victor Hugo était décédé en 1871 et François Victor en 1873. Le portrait de François Victor est conservé au dépôt d'Ivry.

69. Paul Meurice envoie à Ernest Barrias une liste de noms afin de représenter les témoins du drame : Victor Hugo, Victor Schoelcher, Jules Favre, Hippolyte Carnot, de Flotte, Mordier, de Montjean, Michel de Bourges, M. de la Drôme. Lucie Barrias, épouse du sculpteur, lui répond que son mari ne peut tous les représenter. Il a mis en scène les trois premiers; elle lui demande de choisir deux autres noms. Arch. de la maison de Victor Hugo, dossier : Monuments I - Barrias (Correspondance Barrias, correspondance Allar, atelier), lettre de Lucie Barrias à Paul Meurice, 13 février 1901.

70. Arch. de la maison de Victor Hugo, dossier Monuments I - Barrias (Localisation du monument, correspondance Pascal, affaire de la palissade), lettre de Jean-Louis Pascal à Paul Meurice, 10 août 1902.

71. En effet le motif de la statue principale de Hugo, songeur, avec la tête appuyée sur la main gauche et le bas droit replié donnant appui au coude de la main gauche, était le même (avec inversion de la droite et de la gauche) que dans le bas-relief: "Victor Hugo et ses personnages " que Meurice voulait placer devant. De même, la pieuvre s'étalant au pied du poète, allusion dans Les Travailleurs de la mer à Gilliat et la pieuvre, était reprise dans le bas-relief représentant Victor Hugo entouré des personnages de ses romans. 
de mettre sur le devant le bas-relief mouvementé, coloré, bien à l'échelle, de l'enfant mort ${ }^{72}$.

Jean-Louis Pascal proposa une disposition provisoire de ces bronzes permettant de les retirer et de prendre parti ensuite ; Barrias voulut poser tout de suite le basrelief qu'il préférait ; Jean-Louis Pascal essaya de temporiser : "Nous finirons bien par un accord à force de bonne volonté réciproque ${ }^{73}$. Le jour où l'on dévoila au comité le monument (ill. 4), c'est le bas-relief de Victor Hugo et ses personnages, celui que Paul Meurice désirait sur la face, qui apparaît sur les photos. On a donc procédé à une inversion par la suite (ill. 1).

\section{Polémique sur la construction}

Cette polémique opposant Paul Meurice et Ernest Barrias sur le programme du monument fut doublée d'une autre polémique sur sa construction, ralentissant les travaux et entrainant un surcoût dont les deux hommes se renvoyèrent la charge, Jean-Louis Pascal jouant à nouveau le rôle d'arbitre entre les deux parties. En effet, alors qu'en 1899 le coulage des fondations avait commencé, on décida de construire une station de métro sous la place, rendant obligatoire la consolidation de ces dernières $^{74}$. Il fallait d'autre part détruire la fontaine qui ornait le centre de la place ${ }^{75}$ :

« Pour obtenir davantage, c'est-à-dire la participation de la Ville à d'autres travaux sous le monument même, j'entends sous son axe, il faudrait que le jet d'eau actuel fût démoli et que les substructions actuelles restassent deux ans et plus à découvert. C’est inadmissible. Je rouvrirai les démarches [...] Au reste je me tiendrai au courant des agissements du métropolitain et dès cette semaine je descendrai dans la galerie en cours ${ }^{76}$.

Jean-Louis Pascal va se débattre avec la ville de Paris, d'autant plus que le refus par celle-ci d'autoriser l'affichage publicitaire sur la palissade entourant le chantier, entraîna pour l'architecte une perte réelle de ressources. L'architecte était très inquiet

72. Arch. de la maison de Victor Hugo, dossier Monuments I - Barrias (Localisation du monument, correspondance Pascal, affaire de la palissade), lettre de Pascal à Paul Meurice, 14 février 1902.

73. Ibid., pneumatique de Jean-Louis Pascal à Paul Meurice, 16 février 1902.

74. "Vous avez demandé que la ville de Paris prenne des mesures, en vue de donner une résistance suffisante au pied droit de la station du métropolitain avenue Victor Hugo, pour appuyer les fondations du monument à élever à Victor Hugo [...] En conséquence, il sera procédé à la substitution d'une plate-forme horizontale à la plate-forme inclinée prévue pour constituer la base de la culée ». Arch. de la maison de Victor Hugo, dossier Monuments I - Barrias (Localisation du monument, correspondance Pascal, affaire de la palissade), Paris, lettre du directeur administratif de la Voie publique et des Eaux et Égouts, E. Lefrance à l'inspecteur général, 18 mars 1899.

75. Formée de deux bassins concentriques, elle avait donné son nom à la place après 1830 : "rondpoint des bassins".

76. Arch. de la maison de Victor Hugo, dossier Monuments I - Barrias (Localisation du monument, correspondance Pascal, affaire de la palissade), lettre de Jean-Louis Pascal, probablement à Paul Meurice, Paris, le 20 mars 1899. 
pour son monument: "Les travaux du chemin de fer métropolitain ont pénétré sous le terrain qui nous avait été concédé, compromettant la sécurité, la stabilité des parties accessoires de notre monument, et ajoutant à cet inconvénient celui de la trépidation pour tout l'ensemble $"{ }^{77}$. Il craignait de ne pouvoir respecter les délais qu'il s'était engagé à respecter, délais qui prévoyaient une inauguration correspondant au centenaire de la naissance de l'écrivain ${ }^{78}$. Il craignait aussi de dépasser le budget prévu : "L'erreur de faire passer le métropolitain sous le monument me donne les plus graves soucis pour les fondations et détruit mes prévisions budgétaires dans l'évaluation que j'avais faite à Barrias pour cette partie de l'opération ${ }^{79}$. Au printemps 1901, un problème d'infiltration d'eau sur le métropolitain suspend les travaux ${ }^{80}$; Ernest Barrias écrit à Paul Meurice : "Voilà bientôt six mois de perdus, les travaux de fondation sont complètement arrêtés ${ }^{81}$. L'architecte estimait que le surcoût devait en être payé par la Ville de Paris ou le Métropolitain ${ }^{82}$. N'ayant pas obtenu satisfaction, il proposa de "partager les frais ${ }^{83}$ de ce mécompte, qu'il était injuste de laisser à une seule personne laquelle n'en pouvait mais » ${ }^{84}$ :

"C'est du Mont-Dore [station thermale du Puy-de-Dôme] que m'arrive une lettre dans laquelle mon vieux camarade [Barrias] ne se soucie plus de reprendre ce sujet [Victor Hugo et ses personnages] en bas-relief que, par un double changement, on placerait sur la face principale du monument à Victor Hugo. Il me parle de nouveau de l'injustice qu'il y a à lui faire supporter une dépense que rien ne pouvait lui faire prévoir [...] de sa répugnance à demander à l'Administration municipale quelque chose qui ne devrait pas le concerner, alors que le comité et son président pouvaient et devaient éle-

77. Arch. de la maison de Victor Hugo, dossier Monuments I - Barrias (Correspondance Barrias, correspondance Allar, atelier), lettre de Jean-Louis Pascal à Émile Blémont, 21 janvier 1901.

78. "Paul Meurice a raison. En 1900, l'inauguration se confondrait peut-être avec les fêtes de l'Exposition. En 1902, il y a une idée plus grande, plus prenante : le centenaire». Ibid., lettre de Jules Clarétie, Administrateur général de la Comédie-Française, Viroflay, 16 juillet 1896.

79. Ibid., lettre de Jean-Louis Pascal à Paul Meurice, 4 avril 1901.

80. Ibid., lettre de Jean-Louis Pascal à Paul Meurice, 17 mai 1901.

81. Ibid., lettre d'Ernest Barrias à Paul Meurice, 13 juin 1901.

82. "[Il faut] réclamer au préfet de la Seine, à titre de compensation au préjudice causé par cette erreur, une participation à la dépense qui en sera le résultat ». Arch. de la maison de Victor Hugo, dossier Monuments I - Barrias (Correspondance Barrias, correspondance Allar, atelier), lettre de JeanLouis Pascal à Émile Blémont, 21 janvier 1901.

83. « Mon devis portait 7799,22 fr. Ajoutons-y un 10 d'imprévu, soit 779,92 fr. Total : 8 579,14 fr. Il a été dépensé 19 730,88fr. Différence : 11 151,74 fr. Laissons de côté les honoraires d'architecte [...] J'en pose le principe en laissant à une entente le soin d'en fixer le taux. Il semble difficile qu'on puisse en contester le bien-fondé. C'est ma conviction d'architecte et en quelque sorte d'arbitre ». Arch. de la maison de Victor Hugo, dossier Monuments I - Barrias (Correspondance Barrias, correspondance Allar, atelier), lettre de Jean-Louis Pascal à Paul Meurice, Paris, le 23 juin 1902.

84. Arch. de la maison de Victor Hugo, dossier Monuments I - Barrias (Localisation du monument, correspondance Pascal, affaire de la palissade), lettre de Jean-Louis Pascal à Paul Meurice, 19 juillet 1902. 
ver des protestations et des réclamations, quand ils ont été avertis [...] combien il se sent injustement traité, il préfererait abandonner ce qu'il considère comme son droit, que de continuer un débat qu'il qualifie sévèrement " ${ }^{85}$.

Le comité et son président considéraient qu'ayant été mis constamment à l'écart pour tout ce qui touchait à l'exécution du monument, ils n'avaient pas non plus à intervenir, ainsi que l'affirmait Barrias, auprès du Métropolitain ou de l'Administration :

«Des avis des plus compétents et des plus honorables que j’ai cru devoir prendre nous confirment qu'en droit nous ne devons absolument rien. En refusant la compensation doublement utile que je lui offrais, en toute courtoisie, sur d'autres fonds que les fonds épuisés du monument, M. Barrias croit pouvoir qualifier sévèrement notre appréciation très sincère et coupe court lui-même à tout autre arrangement amical ${ }^{86}$.

Pascal défendit vivement Barrias, rappelant que celui-ci ne pouvait être au courant de toutes ces évolutions lorsqu'il avait rédigé son devis :

« Je n'aurais vraiment qu'à laisser tomber toute cette polémique dont l'ami Barrias [...] finit par payer les frais, si je ne devais cependant vous faire observer [...] que vos souvenirs vous servent mal quand vous croyez n'avoir pas été appelé à intervenir auprès de la Ville et du Métropolitain à propos de l'invasion de celui-ci sous nos fondations [...] J'ai une lettre entre autres sous les yeux, que je vous ai communiquée, dans laquelle on me disait que nous aurions dû protester quand la Ville avait autorisé le Métropolitain à passer sous le monument en lui octroyant le même terrain qu'à nous. Comment l'aurions-nous pu deviner ? C'est en 1899 que les faits se passaient. Tout ceci est oiseux, et je n'y insiste que pour vous affirmer l'entière bonne foi de Barrias, bonne foi dont son caractère même est le plus sur garant " ${ }^{87}$.

Pour finir, Paul Meurice et le sculpteur se partagèrent les frais supplémentaires ${ }^{88}$.

Des délégations du monde entier devaient assister à l'inauguration du monument, à laquelle étaient aussi invités des représentants de tous les corps constitués français. Une tribune devait être installée pour les orateurs prononçant des discours au nom du gouvernement, du Sénat, de l'Académie Française, de la Société des Gens de Lettres, du conseil municipal de Paris, du conseil municipal de Besançon ${ }^{89}$.

85. Ibid., lettre de Jean-Louis Pascal à Paul Meurice, 10 août 1902.

86. Ibid., lettre de Paul Meurice, Veules-les-Roses, 3 septembre 1902.

87. Ibid., lettre de Jean-Louis Pascal à Paul Meurice, 8 septembre 1902.

88. June Hargrove, Les Statues de Paris, la représentation des grands hommes dans les rues et sur les places de Paris, op. cit., p. 170.

89. Arch. de la maison de Victor Hugo. Dossier Monuments Barrias (Boîte Centenaire), "Le Centenaire d'Hugo ", L'Écho de Paris, 15 décembre 1901. 
Quelle fut la fortune critique de ce monument? L'extravagance du monument aurait valu à Barrias, le dédain de la postérité ${ }^{90}$. On parla d' " exubérance baroque " ${ }^{91}$. Le bouillonnant journaliste Henri Rochefort (1831-1913), celui que Victor Hugo dépeindra comme : "L'archer fier, le puissant sagittaire, dont la flèche est au flanc de l'Empire abattu " ${ }^{92}$, qui avait dû lui aussi s'exiler à Bruxelles ${ }^{93}$ dans les années soixante, où la famille Hugo l'avait accueilli, reprocha à Ernest Barrias ${ }^{94}$ d'avoir représenté dans son monument l'homme jeune, d'avant l'exil, et non le vieux poète du portrait de Léon Bonnat. Un article du journal L'Éclair disait :

«Ce n'est pas, comme on serait tenté de le croire, le Victor Hugo que nous avons connu dans ses dernières années, que nous serons appelés plus tard à contempler ; c'est au contraire, le Victor Hugo jeune et militant, le front couronné de longs cheveux, les joues entièrement rasées. Autrement dit, c'est le Père et non l'Aïeul que Barrias a voulu représenter » ${ }^{95}$.

Le Journal Les Marges organisa un référendum pour choisir la statue la plus laide de Paris et le Gambetta fut vainqueur. Le Journal des Débats écrivit : "Soyez sûrs, en effet, que le Victor Hugo de Barrias mériterait de lui disputer le prix " 96 . L'Écho de Paris écrivait : "Ce monument sera certainement, avec le monument du Triomphe de la République l'un des plus considérables qui aient été érigés à Paris; il aura du reste demandé à Barrias six années de travail " ${ }^{97}$. Cela ne suffit pas à assurer sa postérité. La statue fut fondue par les Allemands dès le 20 novembre 1941, sans que la décision ait été soumise au secrétaire d'État à l'Éducation nationale et alors même que venait d'être promulguée la loi du 11 octobre 1941 qui permettait ce recours en cas de statue de "gloire nationale " ${ }^{98}$. Quelques années plus tard, on pensa à remplacer le monument par la statue de Victor Hugo que Rodin avait créée pour le Panthéon ${ }^{99}$. Conçue pour être placée le long d'un mur, dans une atmosphère de recueillement, elle ne pouvait garnir une vaste place, servant de cirque à voitures

90. June Hargrove, Les Statues de Paris, la représentation des grands hommes dans les rues et sur les places de Paris, op. cit., p. 171.

91. Ibid., p. 238.

92. Cité par Pierre Milza, Napoléon III, Paris, Perrin, 2004, p. 551.

93. À la suite de l'interdiction de son journal satirique, La Lanterne, qui calomniait la famille impériale, et d'une condamnation à un an de prison et 10000 fr. d'amende. Ibid., p. 550-551.

94. Dans un article intitulé : "La barbe du poète" dans L'Intransigeant du 28 février 1902, journal d'extrême-droite dont Henri Rochefort, jadis appelé le "marquis rouge ", prend la direction à la fin des années 1880 .

95. Arch. de la maison de Victor Hugo. Dossier Monuments Barrias (Presse). «Une statue à Victor Hugo ", L'Éclair, 18 décembre 1897.

96. Journal des Débats, 19 février 1919, cité dans June Hargrove, Les Statues de Paris, la représentation des grands hommes dans les rues et sur les places de Paris, op. cit., note 302, p. 369.

97. Arch. de la maison de Victor Hugo. Dossier Monuments Barrias (Boîte Centenaire), « Le Centenaire d'Hugo ", L'Écho de Paris, 15 décembre 1901.

98. Chantal Martinet, "Les hommages publics », La Gloire de Victor Hugo, op. cit., note 127, p. 297.

99. Elle sera inaugurée dans les jardins du Palais-Royal le 30 septembre 1909, retirée dès 1933 et déposée au musée Rodin de Meudon. Ibid., p. 318. 
automobiles, et dont le centre était visible de partout et de loin. Le projet en fut abandonné ${ }^{100}$.

Ernest Barrias avait tout de même reçu, en 1902, le "prix Lheureux " ${ }^{101}$ pour son monument, prix dont disposait chaque année la Ville de Paris pour récompenser la plus belle œuvre alternativement d'un sculpteur et d'un architecte.

\section{Le monument à Sadi Carnot (1895-1896)}

En 1895, Jean-Louis Pascal réalisa à Bordeaux, en collaboration avec le sculpteur Louis Ernest Barrias, un monument au président de la République Sadi Carnot $(1837-1894)^{102}$, assassiné par l'anarchiste Caserio ${ }^{103}$ à Lyon. Cet assassinat provoqua une grande émotion et donna lieu partout en France à de multiples souscriptions destinées à élever des monuments commémoratifs. Il y eut des souscriptions ouvertes à Lyon, à Dijon (Sadi Carnot avait été député de la Côte-d'Or et conseiller général de 1871 à 1887), à Beaune, à Limoges, sa ville natale, et au total, dans plus de cent cinquante communes. Bordeaux était la première ville que Sadi Carnot avait visitée au début de sa législature. Dès le lendemain de son décès, une souscription ${ }^{104}$ y est ouverte dans les locaux de La Gironde et de La Petite Gironde. Elle atteint rapidement quarante-deux mille cinq cents francs. Ernest Barrias, chargé de l'érection du monument, moyennant le prix de cinquante-sept mille francs ${ }^{105}$, choisit Jean-Louis Pascal ${ }^{106}$ pour le seconder. La direction des travaux était assurée par l'architecte municipal Flandrai.

100. «Le monument de Barrias, qui ornait la place Victor Hugo, à Passy, et qui a été envoyé à la fonte par les Allemands, doit être remplacé. Il n'est paraît-il plus question de dresser une nouvelle statue du grand poète sur cette même place, à cause de la circulation devenue intense dans ce quartier ". Frédéric Saisset, "La fontaine Victor Hugo ", coupure de presse. Arch. de la maison de Victor Hugo. Dossier Monuments Barrias (Remplacement).

101. Décerné pour la première fois, en 1900, à Jules Dalou (1838-1902), pour son Triomphe de la République de la place de la Nation. Charles Girault l'obtint en 1901 pour son Petit Palais. "Ville de Paris, le prix Lheureux ", L'Architecture, 2 janvier 1904.

102. Marie François Sadi Carnot, fils de Lazare Hippolyte Carnot, est né à Limoges le 11 août 1837. Entré à l'Ecole polytechnique en 1857, il est d'abord ingénieur en Savoie. En 1871, il est nommé préfet de la Seine-Inférieure, de l'Eure et du Calvados, puis député à l'Assemblée nationale. Ministre des travaux publics, puis ministre des finances, il est élu président en 1887 et assassiné à Lyon le 24 juin 1894. A.M. Bordeaux, 3016 M 1, La Gironde, 26 juin 1894.

103. Santo-Girolamo Caserio est né le 3 septembre 1873 à Motta Visconti, à $30 \mathrm{~km}$ de Milan, d'Antoine Caserio et de Martine Broglia. Apprenti boulanger à Milan, il se lie avec des anarchistes et est arrêté pour atteinte à l'ordre public. Il s'enfuit à Lugano en Suisse, puis en France, où il met à exécution ses projets d'assassinat du président, à Lyon. A.M. Bordeaux, 3016 M 1, Le Nouvelliste, $1^{\text {er }}$ août 1894 .

104. Comité de la souscription : président : le maire Camille Cousteau, vice-président: Plumeau conseiller général, trésorier: Gounouilhou directeur de La Gironde, secrétaire : Ernest Toulouze rédacteur à $L a$ Gironde.

105. L'État rajoutant une subvention égale au dixième de la dépense totale, soit cinq mille sept cents francs, il ne restait plus qu’à pourvoir à une insuffisance de sept mille francs pour ériger le monument.

106. Honoraires dus à Pascal pour le monument à Carnot : 721,00 fr. 
Le monument se dressait au centre de l'ancienne place Richelieu, actuelle place Jean-Jaurès ${ }^{107}$, en bordure de la Garonne, le long du quai Louis XVIII (ill. 8). L'édicule était ceint d'une belle grille circulaire en fer forgé ${ }^{108}$; quatre candélabres ${ }^{109}$ y fournissaient l'éclairage. Le monument mesurait sept mètres de haut à partir du terre-plein. Il se composait d'une stèle en granit rosé d'Écosse reposant sur un emmarchement en granit gris. Le président Carnot était debout sur la stèle, la main gauche sur le cœur, la main droite appuyée sur un petit socle ${ }^{110}$, dans une pose qui lui était familière. Une grande figure allégorique de l'Histoire était assise sur la partie du socle faisant face à la Garonne ; elle tendait à Carnot la palme dorée des martyrs ${ }^{111}$. Sur le côté gauche du monument, un jeune enfant tenant un bouquet d'immortelles dont quelques fleurs étaient éparses autour de lui, était assis devant un cartouche portant les armes de Bordeaux. Les statues étaient en bronze ${ }^{112}$. Sur le piédestal était gravée cette simple inscription : "À Carnot, président de la République, 1887-1894 ». L'Histoire soutenait de la main gauche une table également en bronze sur laquelle était écrite cette phrase extraite du discours prononcé à Bordeaux le 28 avril 1888 par le président Carnot : "Je suis ici sur la terre classique de la Liberté et le cœur de la population girondine proteste contre tout ce qui pourrait servir les intérêts ou encourager les espérances des ennemis de la République ». Le Petit

107. Dédiée au célèbre tribun (1858-1914), cette place proche des quais fut place Richelieu et auparavant, place du Marché-aux-vins. C’est en effet là qu'on vendait jadis le vin par barriques. Roger Caly, Les rues de Bordeaux des origines à nos jours. Dictionnaire historique et biographique, éd. Princi Negre, 1998, p. 99.

108. La grille en fer forgé, de 5,10 m de diamètre était composée de barreaux en fer rond, avec quatre traverses dans la hauteur du fer. La frise inférieure comprenait une série d'enroulements formant volutes en fer, la frise supérieure comprenait une série de cercles fixés à chaque point de tangence ; les barreaux ronds étaient terminés par des culots avec rinceaux en acier coulé. Elle était fermée par une porte à deux vantaux en fer forgé dont chaque bouclier était gravé du chiffre entrelacé : "CC ». A.M. Bordeaux, 3016 M 1, mairie de Bordeaux, érection du monument Carnot, place Richelieu, ferronnerie et lettre de Pascal au maire, 20 novembre 1895.

109. C'est Charles Gautier, fabricant de bronzes, 10 cours de l'Intendance, à Bordeaux, qui fait les quatre candélabres pour $1200 \mathrm{fr}$. l'un, plus $45 \mathrm{fr}$. pour chacune des seize branches des candélabres, soit 720 fr., car il a fallu modifier les bras des candélabres - trop peu résistants - dessinés par Pascal. Les lanternes sont en bronze, les branches des candélabres en cuivre et bronzées, le corps des candélabres, en fonte de fer.

110. Socle où sont gravées les initiales de la République : "R.F." qui suscita ce commentaire : "La figure placée au bas du piédestal séduit par son allure vraiment artistique, la petite table de nuit colloquée auprès du président de bronze fait sourire ». A.M. Bordeaux, 3016 M 1, "Inauguration de la statue de Carnot ", Le Nouvelliste, 14 septembre 1896.

111. "Pour précéder l'escalier par lequel serait censée monter la figure allégorique pour s'asseoir sur le banc lié au piédestal, je prévois une ligne de pierre ou de marbre constituant pour ainsi dire son chemin dans le tertre de gazon, et partant de l'ouverture pratiquée dans le bahut de la grille pour qu'on puisse aller nettoyer à l'intérieur ". A.M. Bordeaux, 3016 M 1, lettre de Pascal, 3 décembre 1894. Le 5 mars 1912, le conseil municipal décide de faire réparer la palme cassée à l'occasion d'une manifestation, par la maison Leprince et $\mathrm{C}^{\text {ie }}$ de Bordeaux, successeur de la maison Gautier, pour $750 \mathrm{fr}$. Les modèles en plâtre en avaient été détruits sur l'ordre de Barrias, à l'époque décédé.

112. Elles furent fondues par la maison G. Leblanc-Barbedienne, 30 boulevard Poissonnière, Paris. A.M. Bordeaux, 3016 M 1, lettre de Pascal au maire, 16 juin 1896. 


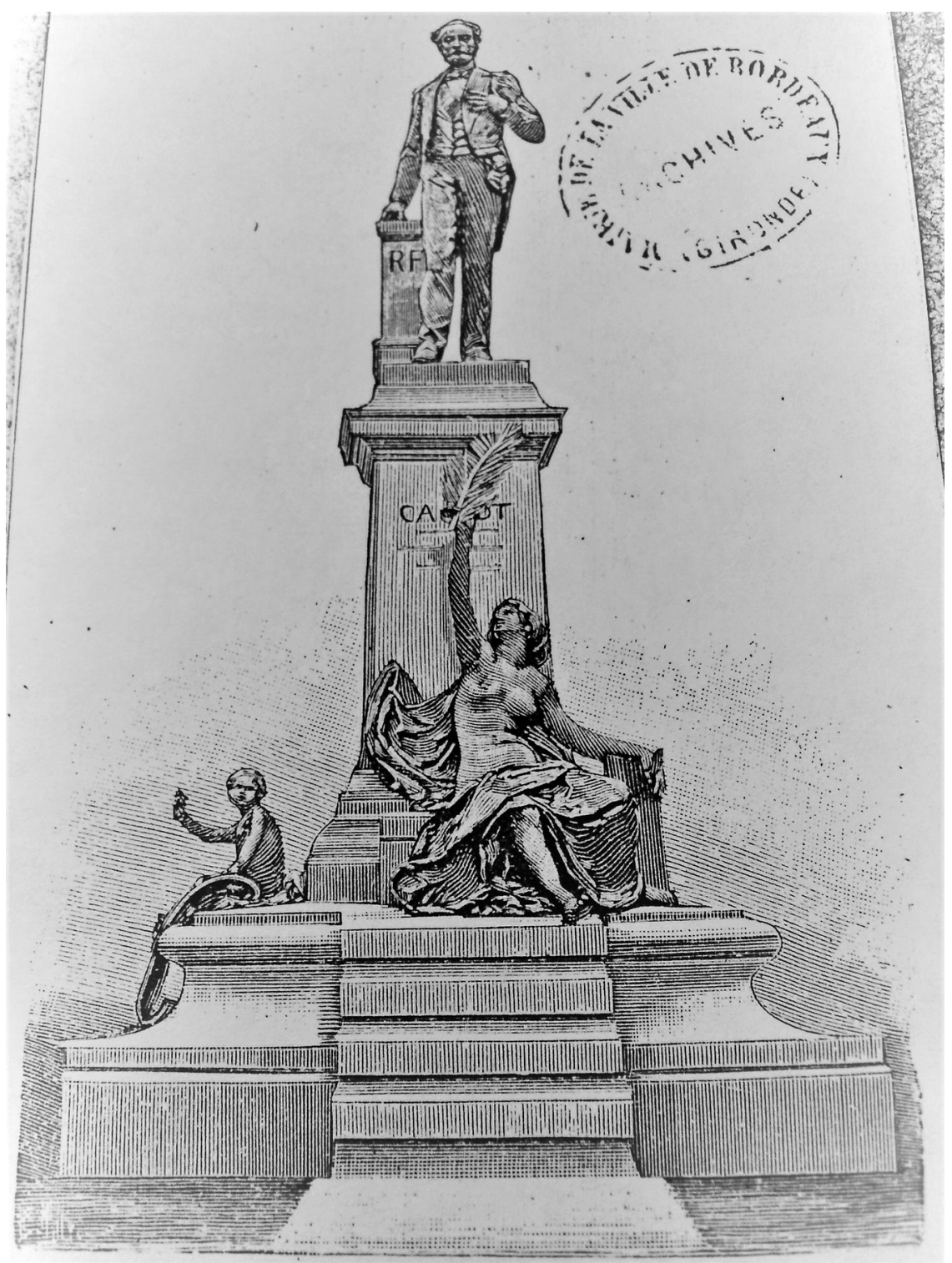

Ill. 8: Le monument de Sadi Carnot à Bordeaux. Jean-Louis Pascal. 1895-1896. Place Richelieu (actuelle place Jean-Jaurès), le long du quai Louis XVIII. Sculpteur Louis Ernest Barrias. Archives municipales de Bordeaux, XXI-75. 
Journal la décrivit ainsi : "Cet ensemble est d'une grande simplicité, est d'un effet réellement imposant. L'œuvre fait le plus grand honneur au maître Barrias et à l'architecte Pascal qui lui a prêté le concours de son talent fin et délicat " ${ }^{113}$.

Jean-Louis Pascal avait réalisé le socle et le piédestal de pierre qui supportait la statue ainsi que le motif de la grille et des candélabres. La ville prenait à sa charge les travaux de terrassement, maçonnerie, entraînés par la construction du piédestal, le coût de la grille et des candélabres entourant le monument, ainsi que les dépenses non couvertes par la souscription ${ }^{114}$.

Pour l'inauguration, on dressa face au monument la tribune destinée au quatorze juillet. Les quatre angles de la place étaient ornés de quatre grands mâts avec trophées de drapeaux et écussons. À chaque mat flottait une riche oriflamme avec cordons et glands dorés. La statue du président était recouverte d'un drapeau tricolore recouvert d'un voile de crêpe, que l'on fit glisser le jour de l'inauguration, le 13 septembre 1896 à dix heures, en présence du ministre de la justice Jean-Baptiste Darlan (1848-1912), aux accents de l'Hymne des Girondins ${ }^{115}$.

\section{Le monument dédié à Charles Garnier (1898-1903)}

Pour Charles Garnier - patron pour lequel il avait toujours gardé un respect attendri ${ }^{116}$-, Jean-Louis Pascal exécute en 1902 un monument "qui possède le mouvement baroque, le luxe coloré, l'abondance décorative du voisin Opéra " 117 (ill. 9). Très vite après la mort de Charles Garnier ${ }^{118}$, la Société centrale des architectes français, dont Garnier avait été le président pendant six ans ${ }^{119}$, décide de lan-

113. A.M. Bordeaux, 3016 M 1, "Le monument à Carnot ", Le Petit Journal, 14 septembre 1896.

114. Le devis pour les travaux de terrassement, fondations, remplissage en maçonnerie de la base, mise en place de la grille et des candélabres s'élevait à $18100 \mathrm{fr}$. C’est la maison F. Liégaux fils, 17 rue du Manège à Bordeaux qui réalisa la grille. La maison Bricard fut chargée de l'exécution des serrures, crémones. Les travaux de terrassements et maçonnerie furent réalisés par Lamire Frères, 73 rue de Soissons, Bordeaux. La maison Bertrand Hauret, 1 place de la Monnaie à Bordeaux a fait aussi des travaux de maçonnerie (maçonnerie en pierre de taille de Bourg pour massifs entre les granits du socle). A.M. Bordeaux, $3016 \mathrm{M} \mathrm{1}$, devis définitif de Barrias pour le monument à Carnot, 8 décembre 1894 .

115. Ibid., lettre du 2 septembre 1896 de l'adjoint délégué à M. Larrégien.

116. Jean-Louis Pascal, "Charles Garnier, architecte de l'Opéra de Paris ", notice lue à l'Assemblée générale de la Société centrale des architectes français, jeudi 27 avril 1899, Paris, Impression Dumoulin.

117. Louis Hautecour, Histoire de l'architecture classique en France, la fin de l'architecture classique (1848-1900), t. VII, Paris, Picard, 1957, p. 433-435.

118. Survenue dans la nuit du 2 au 3 août 1898. Jacques-Maurice Poupinel, "Un monument à Charles Garnier " et "Obsèques de Charles Garnier ", L'Architecture, 1898, $11^{\mathrm{e}}$ année, $\mathrm{n}^{\mathrm{o}} 33$, samedi 13 août 1898, p. 289-293. Les obsèques de Charles Garnier furent célébrées le 6 août, à midi, à Saint-Séverin, sa paroisse. p. 290.

119. Il fut président de la S.C.A.F. en 1889-91 et 1895-97. "Charles Garnier ", L'Architecture, 1898, $11^{\mathrm{e}}$ année, $\mathrm{n}^{\mathrm{o}} 32$, samedi 6 août 1898 , p. 281. 


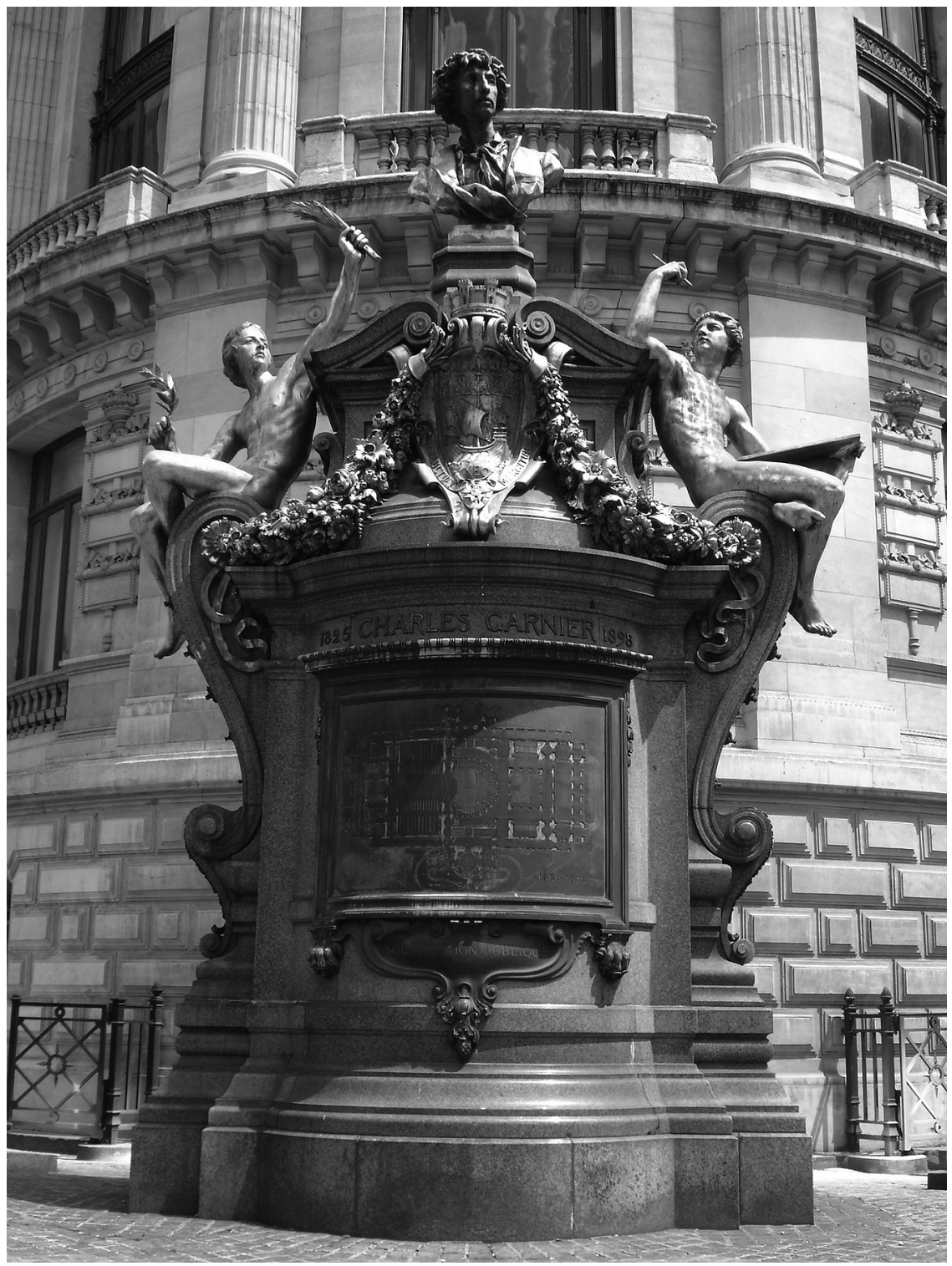

Ill. 9 : Monument à Charles Garnier. Jean-Louis Pascal. 1903. Angle des rues Auber et Scribe, Paris $\left(9^{\mathrm{e}}\right)$. Architecte : Jean-Louis Pascal, sculpteurs : Jean-Baptiste Carpeaux (buste), Gabriel Thomas (la Gloire et le Dessin), Gustave Germain (sculpture décorative), Maison Christofle (plan). Granit d'Écosse, bronze et bronze doré. Cliché auteur. 
cer une souscription destinée à lui élever un monument ${ }^{120}$. Le 12 août 1899 , la souscription avait atteint la somme de 53261,50 francs $^{121}$. Les directeurs de l'Opéra de Paris de l'époque, Eugène Bertrand ${ }^{122}$ et Pedro Gailhard ${ }^{123}$ (1848-1918), proposent que la répétition générale de La Burgonde, opéra de Paul Vidal (18631931) écrit sur un livret d'Émile Bergerat (1845-1923) et Camille de Sainte-Croix (1859-1915) soit jouée le 20 décembre au profit de la souscription au monument ${ }^{124}$. Jean-Louis Pascal fait lui-même un don de deux cents francs tandis que cinquante et un élèves de son atelier souscrivent pour un montant de deux cent treize francs ${ }^{125}$. En 1901, Pascal trouve que le produit de la souscription n'est pas élevé : "C'est entre architectes seulement que la souscription s'est produite et tout spécialement entre anciens élèves de nos ateliers ${ }^{126}$. La somme n'est pas grosse [...] ». Toutefois, quelques architectes américains comme Walter $\operatorname{Cook}^{127}$ (1846-1916) architecte de New York, président de la Société des Architectes en même temps que de celle des anciens élèves de l'École de Paris ou l'architecte John Merven Carrère (1858-1911), " un architecte américain des plus connus [...] le plus galant homme du monde » ${ }^{128}$, de père français et de mère brésilienne, avec qui Pascal avait fait la traversée du continent américain, avaient fait des dons ${ }^{129}$.

120. "Le monument de Charles Garnier ", L'Architecture, 1898, $11^{\mathrm{e}}$ année, $\mathrm{n}^{\mathrm{o}} 34$, samedi 20 août 1898 , p. 297 . Le bureau et le conseil de la S.C.A.F. votent une somme de 3000 fr. pour la souscription collective de la société : "Le monument à Charles Garnier ", L'Architecture, 1898, $11^{\mathrm{e}}$ année, $\mathrm{n}^{\mathrm{o}} 35$, samedi 27 août 1898 , p. 301. Un comité est formé pour mener ce projet à bonne fin : il constitue son bureau : président d'honneur: Georges Leygues, ministre de l'instruction publique et des Beaux-Arts ; président : Alfred Normand, président de la S.C.A.F. ; vice-présidents : Roujon, directeur des Beaux-Arts, Francisque Sarcey, homme de lettres ; secrétaire : G. Larroumet, secrétaire perpétuel de l'Académie des Beaux-Arts ; trésorier : Bartaumieux, architecte trésorier de la S.C.A.F. Lucien Étienne, "Le monument à Charles Garnier ", L'Architecture, 1898, $11^{\mathrm{e}}$ année, $\mathrm{n}^{\circ}$ 46, samedi 12 novembre 1898 , p. 401-402.

121. La somme se partageait ainsi : 15642,27 francs, montant de la représentation de La Burgonde plus 37570,50 francs pour les souscriptions privées plus 49 francs de la part des architectes et amis portugais, soit un total de 53 261,50 francs. "Le monument à Charles Garnier ", L'Architecture, $1900,13^{\mathrm{e}}$ année, $\mathrm{n}^{\mathrm{O}} 22$, samedi 2 juin 1900 , p. 189.

122. Directeur de l'Opéra de Paris de 1892 à 1899.

123. Directeur de l'Opéra de Paris de 1884 à 1891 et de 1893 à 1907.

124. Jacques-Maurice Poupinel, "Le monument à Charles Garnier ", L'Architecture, 1898, $11^{\mathrm{e}}$ année, $\mathrm{n}^{\circ}$ 52, samedi 24 décembre 1898, p. 461-463.

125. Bourbon, Lowell, Bordier, Orième, Humphreys, Brune, Corte, Gaudinot, Cléricetti, Gayet, Corbett, Barrias, Montel, Boissonnas, Wallon, Gonze, Aubry, Ferret, Robida, Hérault, Nash, Bernard, Davis, Bouvard, Césilly, Biasini, Formigé, Perrin, Duval, Peigné, Sauvage, Bougoin, Dujarric, Fayon, Burckhardt, Carré, Ayres, Farquhar, Viard, Morguet, Emery, Herscher, Gosset, Roffé, Lalanne, Levard, Stettler, Héneux, Collin, Montandon, Guchet. " Le monument à Charles Garnier ", L'Architecture, 1899, $12^{\mathrm{e}}$ année, no 14 , samedi 8 avril 1899, p. 118-119.

126. B.E.B.A., Ms 745, Lettres de l'architecte Jean-Louis Pascal à Louise Garnier, lettre $\mathrm{n}^{\circ} 4,10$ octobre 1901.

127. C'était un des collègues de Pascal lors du jugement du concours pour l'université de Berkeley en Californie.

128. B.E.B.A., Ms 745, Lettres de l'architecte Jean-Louis Pascal à Louise Garnier, lettre nº 7,4 mars 1902.

129. Cook a fait un don de 3283,80 francs et Carrère, de 500 francs. Ils étaient tous deux d'anciens élèves de l'École des Beaux-Arts de Paris. Carrère et Thomas Hastings (1860-1929), également 
Dans une lettre à Louise Garnier, datée du 19 mai 1903, le préfet de la Seine, Justin de Selves ${ }^{130}$ (1848-1934), proposa d'attribuer le nom de Charles Garnier, à une voie située aux environs de l'Opéra ${ }^{131}$. On décida que le monument serait élevé à l'extérieur de l'Opéra, au pied de la rotonde de la bibliothèque, entre les deux rampes primitivement destinées à l'entrée du chef de l'État. Puis, on choisit l'architecte: "Le nom de M. Pascal, membre de l'Institut, est à peine prononcé, qu'il est acclamé ». "M. Pascal mettra dans la conception de son ouvre, en même temps que le talent qui le distingue, tout son cœur d'ami et de collaborateur si apprécié du maître " dit Gustave Larroumet pour vaincre ses scrupules ${ }^{132}$.

Le monument, qui est situé à l'angle des rues Scribe et Auber, est constitué de deux grandes consoles de support librement accolées aux flancs d'un piédestal supportant deux figures allégoriques aux attitudes mouvementées : la Gloire, représentée sous les traits du fils de Garnier, décédé peu après son père ${ }^{133}$, et le Dessin, toutes deux dues au ciseau de Jules Gabriel Thomas, ami de Garnier dès l'école primaire et qui avait remporté le grand prix la même année que lui. Jean-Louis Pascal voyait les deux allégories comme deux « jeunes hommes dont l'un, qui ressemble à son fils, le glorifie et l'autre s'inspire de ses œuvres [...] dorés comme des génies, accompagnant le dieu [Garnier] ». Pour la sculpture de la Gloire, Thomas s'est inspiré de cette pensée : "Le fils offrant à son père la palme que le monde entier lui avait décernée ". "L'éphèbe tenant la palme est plus qu'une figure allégorique quelconque ; sous ce bronze, est enfermée une âme, l'âme du fils de Charles Garnier" notera un critique ${ }^{134}$. C'est Pascal qui avait tenu à ce que Thomas fasse les deux jeunes allégories; il écrit à Louise Garnier :

"Avec le très sérieux artiste auquel nous pensons, [les deux statues] seront toujours dignes de la place; il leur manquera peut-être cette fleur de la jeunesse qu'il faudrait imprimer à l'œuvre entière; mais je crois comme vous qu'il sera bien difficile sans contrister beaucoup ce vieil ami de le priver de cette dernière œuvre ${ }^{135}$."

ancien élève de l'École, vont introduire aux États-Unis les principes qu'ils y avaient appris - le plan fonctionnel et l'utilisation appropriée des matériaux - dès 1888 avec le Ponce de Leon Hôtel à Saint Augustine en Floride. La Bibliothèque publique de New York (concours : 1897) qu'ils construisent entre 1902-1911 est la première manifestation du mouvement "Beaux-Arts" en Amérique. Macmillan Encyclopedia of Architects, Adolf K. Placzek, éd. in chief, Londres, The Free Press, 1982, vol. 1, p. 387-388.

130. Préfet de la Seine, nommé le 23 mai 1896 jusqu'en juin 1911 en remplacement d'Eugène Poubelle.

131. "Le monument à Charles Garnier", suivi de "La rue Charles Garnier ", L'Architecture, 1903 , $16^{\mathrm{e}}$ année, $\mathrm{n}^{\mathrm{o}} 23$, samedi 6 juin 1903 , p. 217.

132. Lucien Étienne, «Le monument à Charles Garnier", L'Architecture, 1898, $11^{\mathrm{e}}$ année, $\mathrm{n}^{\circ} 46$, samedi 12 novembre 1898 , p. 402.

133. Garnier avait eu deux fils: Daniel mort à deux ans d'une méningite et Charles François Paul dit Christian, dit Nino, décédé à 26 ans de la tuberculose, soit un mois après son père. Charles Garnier décède le 3 août 1898, son fils Christian, le 4 septembre 1898. "Christian Garnier ", L'Architecture, $1899,12^{\mathrm{e}}$ année, $\mathrm{n}^{\circ}$ 5, samedi 4 février 1899 , p. $42-43$.

134. G. Rozet, "Le monument à Charles Garnier », L'Architecture, 1903, p. 306.

135. B.E.B.A., Ms 744, Lettres de l'architecte Jean-Louis Pascal à Louise Garnier, lettre n 264,13 novembre 1899. 
Entre les deux volutes où sont assis les génies, le socle s'incurve, orné du plan de l'Opéra ; il est dominé par un écusson aux armes de Paris enguirlandé de fleurs. Celui-ci s'adosse à une demi-stèle placée au-dessus du piédestal, dont le fronton interrompu à enroulements supporte le buste de Garnier ${ }^{136}$ d'après Jean-Baptiste Carpeaux (1827-1875) ${ }^{137}$. Pascal s'était résolu à n'avoir qu'un buste, les contraintes financières l'ayant privé de la statue en pied qu'il souhaitait (ill. 10) :

"Moi seul peut-être continuerai à avoir du regret de ne pas voir mon cher grand-chef dans le laisser-aller de sa tournure élégante, fine, originale, en pied, fier et simple, comme si le bronze l'avait surpris vivant. La résistance de tous avait bien raison peut-être puisqu'il aurait fallu obtenir de l'État une autre commande quand il aura tant de peine à payer les autres ${ }^{138}$. "

Pascal trouvait que le buste manquait d'ampleur :

"Pour le buste et son support, j'ai une retouche à faire à ce dernier qui manque d'ampleur; mais je n'ai pas encore pris mon parti de faire grandir, je ne sais encore comment ni par qui, le buste de Carpeaux, simplement parce que tout le monument prépare la statue; que c'est par des moyens factices, sortant mal de la composition, que je suis obligé de me contenter d'un buste et que j'ai toujours de la peine à me décider à réaliser ce qui est contre mon sentiment [...] je ne vous cache pas que si j'avais été sculpteur, il y a longtemps que j'aurais essayé la nerveuse figure - que j'entrevois sans pouvoir la construire -, pour mettre tout le monde, vous comprise, de mon avis ${ }^{139}$.

Le buste fut en définitive doublé par rapport à sa taille originale, comme le rapporte Pascal à Louise Garnier :

136. Le buste en bronze a été exposé au Salon de 1869, sous le no 3284 . Appartenant à Louise Garnier, il a figuré en 1900 à l'exposition centennale de l'art français, sous le n 1501 . Le marbre, signé et daté de 1869, appartenant aussi à Louise Garnier, a fait partie en 1912 de l'exposition Carpeaux à la salle du Jeu de Paume. Le modèle en plâtre du buste de Garnier est au musée de l'Opéra. Le musée de Valenciennes en possède aussi un exemplaire en bronze donné par l'architecte Moyaux. Un autre exemplaire de ce buste ornait la salle du conseil de l'École des Beaux-Arts à Paris. Stanislas Lami, Dictionnaire des sculpteurs de l'École Française au XIX $X^{e}$ siècle, Paris, éd. Honoré Champion, 1916, vol. 1, p. 269-270.

137. Charles Garnier avait rencontré Jean-Baptiste Carpeaux à l'École de dessin de la rue de l'Écolede-Médecine et était resté toute sa vie très lié avec lui ; il l'admirait et lui confia la réalisation d'un des groupes sculptés de la façade de l'Opéra, La Danse. Il lui dédicacera ainsi l'exemplaire de son ouvrage $\grave{A}$ Travers les arts: "Veux-tu accepter ce petit livre, il te rappellera le souvenir d'un ami qui est tout fier d'avoir servi de modèle pour un de tes chefs-d'œuvre. " Bibl. Opéra, Fonds Garnier, pièce 122, liste des amis de Garnier annotée, 6 p. Ms., numérotées de 1 à 19.

138. B.E.B.A., Ms 744, Lettres de l'architecte Jean-Louis Pascal à Louise Garnier, lettre $\mathrm{n}^{\circ} 269$, Luchon, 17 septembre 1900 .

139. Ibid., lettre $\mathrm{n}^{\circ}$ 271, Paris, le 10 novembre 1900. 


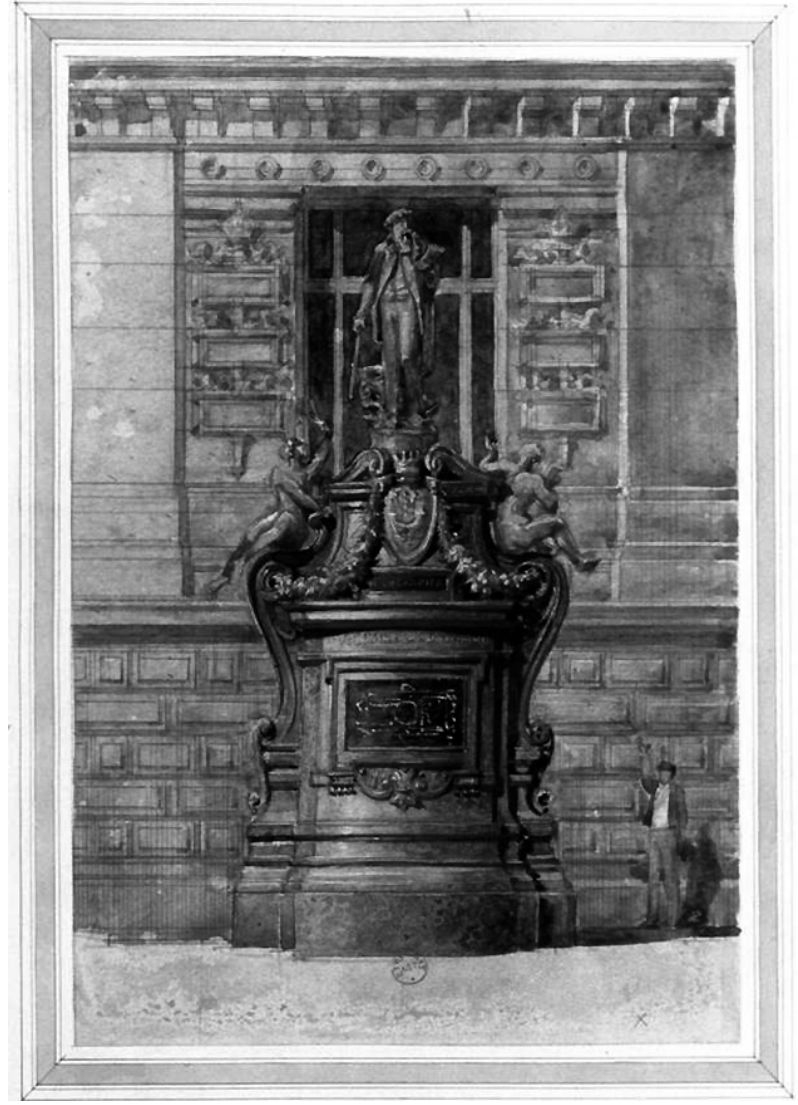

Ill. 10 : Modèle du monument à Charles Garnier proposé par Jean-Louis Pascal, alors qu’il désirait une statue en pied pour représenter le grand architecte. Aquarelle. B.n.F., dp. Est., Ha 132.

"La deuxième visite avec Thomas et le représentant de la maison Christofle, M. Hermel, nous a montré chez le grandisseur, un spécialiste du nom de Haligon, rue d'Alleray, $\left[\mathrm{n}^{\circ}\right]$ 81, qui est aussi un réducteur, le buste double en hauteur, (ce qui fait un cube considérable), de celui de Carpeaux. Le bon juge qu'est Thomas en cette opération toute spéciale et difficile a fait des compliments sincères au praticien; il ne croyait pas à un aussi bon résultat et il y a lieu d'en être satisfait exceptionnellement. Vous ne vous étonnerez pas, chère Madame, que je trouve insuffisant pour la dignité du bronze le détail de la chevelure par exemple, si parfaites que soient les masses, les boucles de cette coiffure destinée à être vue à huit mètres du trottoir. Je n'y mets nul parti pris, cela va sans dire, et puisque Thomas est particulièrement content, comment hésiterais-je ? ${ }^{140}$ Demain je fais contrôler les aplombs du

140. Plus tard, Pascal se félicitera du résultat, comme l'indique une lettre de J.-L. Pascal à Gustave Germain, 12 octobre 1908 aimablement communiquée par les descendants de ce dernier. Il semble tout de même un peu trop petit par rapport à l'ensemble. 
socle pour que l'on puisse emporter le plâtre rue de Bondy où je m'assurerai une dernière fois des concordances avec le granit et on pourra procéder aux opérations de fonte " ${ }^{141}$.

Jean-Louis Pascal utilise la polychromie des matériaux chère à Charles Garnier pour l'Opéra : le piédestal est en granit rouge d'Écosse ${ }^{142}$, choisi pour "sa solide et chaude coloration » que Garnier aimait. Ce dernier l'avait remarqué lors d'un voyage à Londres, alors qu'il construisait l'Opéra : "J'avais remarqué [...] les colonnes de granit qui ornent la façade du Jockey-Club ; ces colonnes fort belles m'avaient paru réunir toutes les conditions de pureté et de poli désirables. J'ai cherché leur provenance [...] le granit provenant d'Aberdeen, en Écosse, est la propriété de MM. Mac Donald, Field et $\mathrm{C}^{\mathrm{ie}}{ }^{143}$. La maison Leclercq et Bourdon assura la mise en ouvre et la pose ${ }^{144}$ : «Notre granitier Leclercq était parti pour l'Écosse [écrit-il à Louise Garnier en 1901] surtout pour s'assurer de l'état d'avancement de notre cher monument. Il en revient enchanté de la beauté de l'exécution et de la matière, surpris de la grandeur du motif, assuré du bon effet à attendre d'un travail où, paraît-il, les exécutants mettent tout leur amour-propre ${ }^{145}$."

Pascal associe la pierre rouge aux bronzes du buste, des allégories et de l'écusson enguirlandé, bronzes qui furent dorés par la suite, ce qui provoqua un tollé ${ }^{146}$; "M. Pascal a voulu que le bronze déjà lié si harmonieusement au granit rouge d'Écosse par le seul jeu des colorations des deux matières se poursuivît en chute sur les consoles de support " ${ }^{147}$. Jean-Louis Pascal hésitait à dorer les bronzes ; il demanda son avis à Henri Bénard dont il était très proche ; celui-ci construisait alors le palais fédéral à Mexico; Pascal lui demanda aussi de trouver là-bas des souscripteurs pour le monument à Garnier ; Bénard le loua pour son monument :

"Tous mes plus chauds et plus sincères compliments, tu as certainement trouvé la note juste. Non, ne dore pas ton buste et les figures; il me semble que le bronze doit mieux se marier à ton granit que des tons d'or. Hélas !

141. B.E.B.A., Ms 745, Lettres de l'architecte Jean-Louis Pascal à Louise Garnier, lettre n' 10, 2 septembre 1902.

142. Le granit vient de la maison Mac Donald, celle-là même qui a fourni les granits pour l'Opéra, A. Dupuis, "L'inauguration du monument de Charles Garnier. Samedi 20 juin 1903 ", L'Architecture, 1903, 16 e année, no 26, samedi 27 juin 1903, p. 255.

143. Bibl. Opéra, fonds Garnier, pièce 128, lettre de Garnier à S.E. le ministre de la maison de l'Empereur et des Beaux-Arts, 31 octobre 1864.

144. Pascal avait déjà travaillé avec Leclercq à Bordeaux pour le monument à Carnot réalisé avec Barrias; il en avait été très satisfait. B.E.B.A., Ms 744, Lettres de l'architecte Jean-Louis Pascal à Louise Garnier, lettre no 269, Luchon, 17 septembre 1900. Il l'emploie aussi au même moment à la Bibliothèque nationale.

145. B.E.B.A., Ms 745, Lettres de l'architecte Jean-Louis Pascal à Louise Garnier, lettre nº 1, 6 juillet 1901.

146. "Monument à Charles Garnier ", La Construction moderne, 1904-1905, p. 278-279.

147. Louis-Charles Boileau, "Causerie", L'Architecture, 1903, p. 296. 
Je voudrais pouvoir te trouver des souscripteurs ici mais je ne crois pas que cela se puisse. Les gens ici sont très égoïstes " ${ }^{148}$.

Le président d'honneur de la chambre syndicale des doreurs ornemanistes, Tardif, proposa de dorer gracieusement le monument ${ }^{149}$; Pascal était tenté :

"L'éclat, le brillant, l'excessif, que mon grand-chef n'avait pas craint lors de l'inauguration de la façade de l'Opéra, [écrit-il à Louise Garnier] ne manqueront pas de susciter les mêmes critiques, mais j'y opposerai l'impassibilité qui fut la sienne alors, bien que peu dans son tempérament et je compte avoir raison comme lui quand auront passé quelques mois d'embarras, de poussière et de rafales sur nos bronzes déjà si fort noircis " ${ }^{150}$.

La presse ne fut pas unanime :

"Comme je m'y attendais, [raconte Pascal à Louise Garnier] je n’ai pas qu'une presse favorable, mais quelques appréciations de personnes compétentes m'en consolent et, de plus, je n'ai pas changé d'opinion [...] Veuillez ne pas vous alarmer de l'éclat offensif actuel des figures, du buste et des armes de la Ville de Paris que je n'ai pas voulu laisser patiner, parce que j'estime que toute patine factice gêne la belle production de la patine normale que se chargent de dorer avec le temps les agents atmosphériques dont nous disposons avec excès dans Paris ${ }^{151}$."

Comme le déclara Jean-Louis Pascal le jour de l'inauguration :

" Rien n'aurait été trop beau, trop coloré, trop riche pour caractériser cet improvisateur dont la fécondité bouillonnante était réglée comme d'instinct par l'arrangement, l'ordre et la composition, si indispensable à nos œuvres, pour fêter cet enthousiaste de la beauté surabondante, cet enivré de la couleur sur de nobles formes ${ }^{152}$.

Les sculptures d'ornements sont dues au sculpteur ornemaniste Gustave Germain ${ }^{153}$ et c'est la maison Christofle qui monta les bronzes fondus par la mai-

148. B.n.F., Mss, N.a.fr. 25093, lettre d’Henri Bénard à Jean-Louis Pascal, Mexico, 18 juillet 1903, p. 17.

149. B.E.B.A., Ms 745, Lettres de l'architecte Jean-Louis Pascal à Louise Garnier, lettre no 15, 28 août 1904.

150. Ibid., lettre $\mathrm{n}^{\circ} 17,4$ janvier 1905.

151. Ibid., lettre n ${ }^{\circ} 18$, Paris, 26 mars 1905.

152. Discours de Jean-Louis Pascal cité par A. Dupuis, "L'inauguration du monument de Charles Garnier. Samedi 20 juin 1903 ", L'Architecture, op. cit., p. 255.

153. "Germain, le sculpteur de ces ornements, était à peine un jeune homme quand il débuta chez Darvant [...] dans les ateliers de décoration de notre grand chantier, et y reçut l'empreinte du maître ». Ibid. Gustave Germain avait collaboré avec Jean-Louis Pascal lors de l'exposition universelle de 1878, lors de la construction de l'hôtel Königswarter en 1881, du tombeau de Jules Michelet en 1882, et surtout sur le chantier de la Bibliothèque nationale. Ce dernier lui écrit : "J'étais déjà bien flottant pour me charger de travaux dans mon état de fatigue et d'énervement et après tous mes malheurs [décès de ses fils et de son père] je comprends que l'heure de la retraite 
son Jabeuf ${ }^{154}$ et grava le plan de l'Opéra de Paris sur l'avant du piédestal : «Le plan représente quelque chose de très spécial dont nos bronziers n'ont pas l'habitude, en sorte qu'il y a eu des tâtonnements, des essais ${ }^{155}$."

Le 2 septembre 1902, Pascal annonce à Louise Garnier : "Le plan est gravé dans le plâtre pour toutes les parties importantes de la construction, pour ce que nous appelons le poché. On va en tirer un galvano dans lequel le graveur déterminera tous les petits traits d'accompagnement : marches d'escalier, lignes de retraite, bases de colonnes, balustrades, etc. " ${ }^{156}$. En dessous du plan, il restait juste la place d'indiquer que le monument était le résultat d'une souscription. La seule place vierge pour indiquer les titres de Garnier se trouvait à l'arrière du monument; on y apposa une plaque de granit vert réalisée en Allemagne sur laquelle ils furent gravés; le texte en avait été rédigé par Louise Garnier ${ }^{157}$.

En août 1902, les allégories de Thomas sont montées :

"Qu'ils n'aient pas la vie, la variété, la chaleur et le mouvement que je leur aurais souhaités, au moins pour l'un d'eux, vous ne l'ignorez pas et le bronze n'y a rien changé mais ils sont fort bien et la richesse de la matière ajoute à leur noblesse ${ }^{158}$.

L'État, voulant participer à l'hommage du grand architecte, avait offert une partie des marbres ou des granits devant entrer dans la composition du monument. Pascal alla donc au dépôt des marbres du quai d'Orsay pour faire son choix : " J'ai demandé [écrit-il à Mme Garnier] à l'État de me donner une dizaine de morceaux de granit ordinaire, que je sais dès longtemps inutilisés au dépôt des marbres pour en faire le socle général sur lequel reposera le granit d'Écosse ${ }^{159}$. " Il jeta son dévolu sur quelques blocs de granit recouverts d'une épaisse couche de poussière, qui, d'après le journal Le Temps, avaient une histoire particulière : au lendemain d'Austerlitz, Napoléon $1^{\text {er }}$ conçut le projet de faire élever la colonne de bronze de la place Vendôme, et commanda en Corse les granits destinés au soubassement. Ceux-ci arrivèrent à Paris au début de l'année 1806 et l'un d'eux fut scellé le 25 août 1806 comme première pierre du monument par Jean-Baptiste de Nompère de Champagny, ministre de l'intérieur ${ }^{160}$; la plupart des autres ayant été employés dans la construc-

a sonné pour moi. Je décide de fermer mon atelier lorsque toutefois j'aurai terminé avec vous les modèles du monument Garnier ». Lettre de Gustave Germain à Jean-Louis Pascal, septembre 1900, appartenant aux héritiers de Gustave Germain, citée par Michel-Gasse (†) dans sa biographie du sculpteur qu'il avait eu la gentillesse de me confier.

154. Jabeuf, 10 rue de l'Asile Popincourt, Paris.

155. B.E.B.A., Ms 745, Lettres de l'architecte Jean-Louis Pascal à Louise Garnier, lettre no 9, 6 août 1902.

156. Ibid., lettre $\mathrm{n}^{\mathrm{o}} 10,2$ septembre 1902.

157. Ibid., lettre $\mathrm{n}^{\circ} 2,8$ juillet 1901 .

158. Ibid., lettre $n^{\circ} 9,6$ août 1902.

159. B.E.B.A., Ms 744, Lettres de l'architecte Jean-Louis Pascal à Louise Garnier, lettre n 269, Luchon, 17 septembre 1900 .

160. Il fut ministre de l'intérieur du 7 août 1804 au 9 août 1807. 


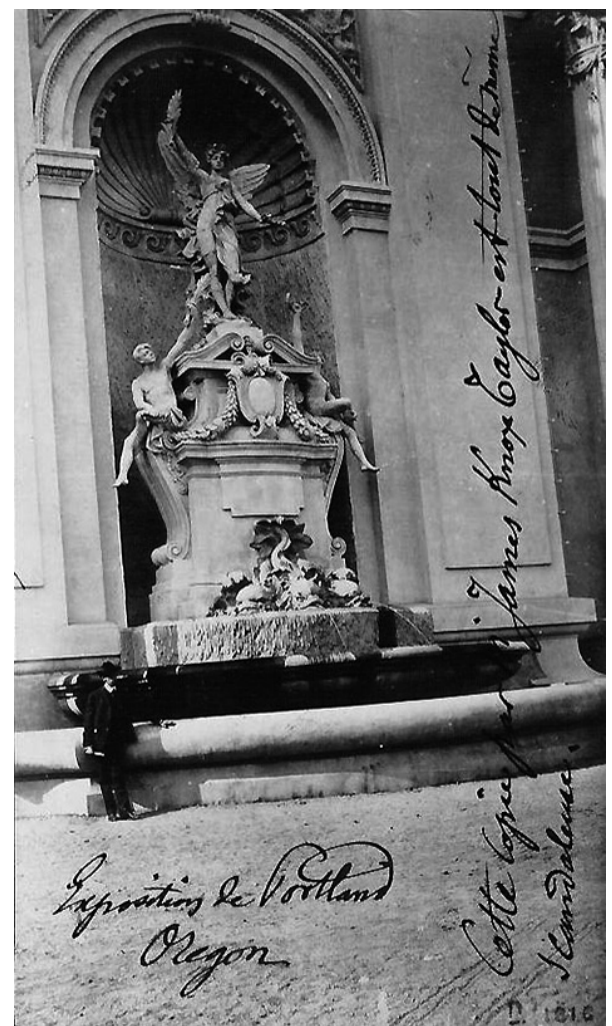

Ill. 11 : Fontaine. Façade du bâtiment principal de l'exposition de Portland dans l'Oregon. États-Unis. 1907. James Knox Taylor architecte. Photo originale de Paul Philippe Cret. B.n.F., dp. Est., Ha 132. Cliché $n^{\circ}$ : 2000-B-161844.

tion du soubassement, quelques-uns demeurèrent sans usage et furent mis de côté : ce sont eux que Pascal fit tailler et transporter rue Auber où ils servirent de soubassement au monument de Charles Garnier ${ }^{161}$. L'inauguration fut fixée au samedi 20 juin 1903, à dix heures du matin. Jean-Louis Pascal fit un discours ${ }^{162}$, ainsi qu'Alfred Normand, président du comité chargé d'ériger le monument, Constant Moyaux, président de la Société centrale des architectes français, Gustave Larroumet (1852-1903), secrétaire perpétuel de l'Académie des Beaux-Arts, et Joseph Chaumié

161. "Nouvelles. Paris. Le monument de Charles Garnier et la colonne Vendôme", La Construction moderne, 1900-1901, 13 juillet 1901, p. 492. Ceci est confirmé par Pascal qui, dans une lettre à Louise Garnier, parle de la "pose de nos morceaux de granit provenant de la colonne Vendôme, ou tout au moins de même origine que le socle de celle-ci. " B.E.B.A., Ms 745, Lettres de l'architecte Jean-Louis Pascal à Louise Garnier, lettre $\mathrm{n}^{\circ} 1,6$ juillet 1901. La direction des Beaux-Arts offrit les bronzes.

162. A. Dupuis, "L'inauguration du monument de Charles Garnier. Samedi 20 juin 1903 ", L'Architecture, op. cit., p. 253-258. 
(1849-1919), ministre de l'instruction publique et des Beaux-Arts ${ }^{163}$. À la fin de la cérémonie d'inauguration du monument, la garde Républicaine joua l'ouverture de Phèdre de Jules Massenet (1842-1912) et la Marche de Déjanire de Camille Saint-Saëns (1835-1921).

En hommage supplémentaire à Garnier, Pascal proposa pour le concours Rougevin de 1902 ce programme : «Le frontispice d'un ouvrage supposé commandé par l'État sur les œuvres et la vie de Charles Garnier ${ }^{164}$."

En 1907, l'œuvre de Jean-Louis Pascal est copiée, lors d'une exposition à Portland dans l'Oregon. James Knox Taylor, architecte officiel du gouvernement, plagie ce monument pour en faire une fontaine (ill. 11). C'est Paul Philippe Cret (1876-1945), ancien élève de Pascal établi aux États-Unis, qui prévient son patron, avec photo à l'appui : "L'original de cette fontaine peut se voir, à deux exemplaires, sur la façade du bâtiment principal de l'exposition de Portland (Oregon). Il est signé James Knox Taylor architecte officiel du gouvernement (ce monsieur est même le seul à jouir de cette distinction) ${ }^{165}$. Et Paul Cret ajoute sur la photo : "Cette copie par M. James Knox Taylor est tout de même scandaleuse ».

163. Il fut ministre de l'instruction publique et des Beaux-Arts du 7 juin 1902 au 24 janvier 1905.

164. B.E.B.A., Ms 745, Lettres de l'architecte Jean-Louis Pascal à Louise Garnier, lettre no 6, 13 février 1902 .

165. Lettre de Paul Cret à Jean-Louis Pascal, 27 mars 1907, B.n.F., dp. Est, Ha 132. 
